\title{
A LOOSELY COUPLED SCHEME FOR FICTITIOUS DOMAIN APPROXIMATIONS OF FLUID-STRUCTURE INTERACTION PROBLEMS WITH IMMERSED THIN-WALLED STRUCTURES*
}

\author{
LUDOVIC BOILEVIN-KAYL ${ }^{\dagger}$, MIGUEL A. FERNÁNDEZ ${ }^{\dagger}$, \\ AND JEAN-FRÉDÉRIC GERBEAU ${ }^{\dagger}$
}

Abstract. Fictitious domain approximations of fluid-structure interaction problems are generally discretized in time using strongly coupled schemes. This guarantees unconditional stability but at the price of solving a computationally demanding coupled system at each time-step. The design of loosely coupled schemes (i.e., methods that invoke the fluid and solid solvers only once per time-step) is of fundamental interest, especially for three-dimensional simulations, but the existing approaches are known to suffer from severe stability and/or time accuracy issues. We propose a new approach that overcomes these difficulties in the case of the coupling with immersed thin-walled structures.

Key words. fluid-structure interaction, incompressible fluid, immersed thin-walled structure, unfitted meshes, fictitious domain method, coupling schemes

AMS subject classifications. 65M85, 74F10, 76M10

DOI. $10.1137 / 18 \mathrm{M} 1192779$

1. Introduction. One of the main difficulties that have to be faced when solving incompressible fluid-structure interaction problems are the numerical issues related to the added-mass effect (see, e.g., [39, 17, 27, 49]). This difficulty has been traditionally overcome by considering strongly coupled schemes, in which the interface conditions are treated in a fully implicit fashion. This ensures stability and time accuracy but at the price of solving a heterogeneous ill-conditioned system at each time-step, which can be computationally demanding in practice.

Over the last decade, significant advances have been achieved in the development and in the analysis of fluid-solid splitting schemes that avoid strong coupling, without compromising stability and accuracy. In the majority of these studies, the spatial discretization is based on body fitted fluid meshes (see, e.g., [21, 44, 5, 14, 31, 13, $20,25,6,26,23,38])$. Fitted meshes are very appealing because they facilitate the accurate prescription of the interface conditions. However, this framework rapidly becomes cumbersome or unfeasible in the presence of large interface deflections or of topological changes (e.g., due to contact between solids). In this case, the alternative is to consider an unfitted mesh formulation, in which the fluid mesh is independent of the solid mesh (see, e.g., [43, 40, 51, 28, 45, 18, 3, 9, 15, 34, 10, 1, 33]).

Within the unfitted mesh framework, splitting schemes which avoid strong coupling are rare in the literature. In fact, we are only aware of the methods reported in $[9,2,36]$, using immersed boundary or fictitious domain methods, and in [15, 1, 33], using unfitted Nitsche based methods with cut-elements. The fundamental drawback of the loosely coupled (or explicit coupling) schemes, reported in $[9,15,33,36]$, is

*Submitted to the journal's Computational Methods in Science and Engineering section June 11, 2018; accepted for publication (in revised form) February 13, 2019; published electronically DATE. http://www.siam.org/journals/sisc/x-x/M119277.html

Funding: This work was supported by the project MIVANA, a collaborative project for the development of new technologies for mitral valve repair, which was led by the start-up company Kephalios, with the participation of the start-up company Epygon, who received funds from the French government, in the context of the program "Investissement d'Avenir."

†Inria Paris, 75012 Paris, France and Sorbonne Université, UMR 7598 LJLL, 75005 Paris, France (ludovic.boilevin-kayl@inria.fr, miguel.fernandez@inria.fr, jean-frederic.gerbeau@inria.fr). 
that their stability/accuracy demands severe time-step restrictions or is limited by the amount of added-mass effect. These issues have been recently circumvented in $[1,2]$, by borrowing the ideas from [20], but at the price of compromising the explicit nature of the coupling scheme. Indeed, the resulting methods are only semi-implicit (see also [22]).

In this paper, we introduce and analyze a new loosely coupled scheme for fictitious domain approximations of fluid-structure interaction problems with immersed thinwalled structures that overcomes the above mentioned issues. Our starting point is the semi-implicit coupling scheme reported in [2]. We show that the combination of an appropriate choice of the Lagrange multipliers space (equivalent to a collocation method) with a mass lumping approximation in the solid yield a loosely coupled scheme. We also present a general stability result that proves that the scheme is unconditionally stable in the energy norm. Numerical experiments in a series of representative twodimensional examples, involving large interface deflections and topology changes in the fluid domain, illustrate the performance of the proposed approach.

The rest of the paper is organized as follows. Section 2 presents the coupled problem considered through the paper. The fictitious domain spatial approximation is introduced in section 3. Section 4 presents the new coupling scheme and its stability analysis. The numerical experiments are reported in section 5. Finally, a summary of the main results obtained with some lines of future research are drawn in section 6 .

2. Problem setting. We consider a fluid-structure interaction problem in which the fluid is described by the incompressible Navier-Stokes equations and the structure by a thin-walled solid model (curved beam in two dimensions or shell in three dimensions). Let $\Sigma \subset \mathbb{R}^{d}$ be the reference configuration of the solid mid-surface $(d=2,3)$. The current position of the interface, denoted by $\Sigma(t)$, is parameterized by its motion map $\phi: \Sigma \times \mathbb{R}^{+} \longrightarrow \mathbb{R}^{d}$ as $\Sigma(t)=\phi(\Sigma, t)$, with $\phi \stackrel{\text { def }}{=} \boldsymbol{I}_{\Sigma}+\boldsymbol{d}$, where $\boldsymbol{d}$ denotes the displacement of the solid. In order to ease the presentation, we introduce the notation $\phi_{t} \stackrel{\text { def }}{=} \phi(\cdot, t)$, so that we also have $\Sigma(t)=\phi_{t}(\Sigma)$. The structure is supposed to move within a domain $\Omega \subset \mathbb{R}^{d}$ with boundary $\Gamma \stackrel{\text { def }}{=} \partial \Omega$ (see Figure 1). For simplicity and without loss of generality, $\Omega$ is assumed to be fixed. The fluid is described in the time-dependent control volume $\Omega(t) \stackrel{\text { def }}{=} \Omega \backslash \Sigma(t) \subset \mathbb{R}^{d}$, with its boundary partitioned as $\partial \Omega(t)=\Sigma(t) \cup \Gamma$. The interface $\Sigma(t)$ is assumed to be oriented by a unit normal vector field denoted by $\boldsymbol{n}^{\mathrm{s}}$. This induces a positive and a negative side in the fluid domain $\Omega(t)$, with respective unit normals $\boldsymbol{n}^{+} \stackrel{\text { def }}{=} \boldsymbol{n}^{\text {s }}$ and $\boldsymbol{n}^{-} \stackrel{\text { def }}{=}-\boldsymbol{n}^{\text {s }}$ on $\Sigma(t)$. For a given continuous scalar or tensorial field $f$ defined in $\Omega(t)$ (possibly discontinuous across the interface $\Sigma(t)$ ), we define

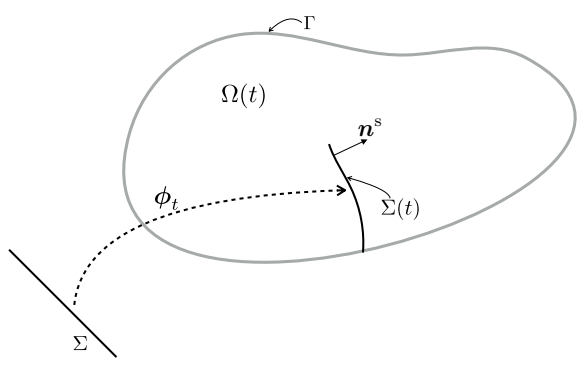

FIG. 1. Geometric description. 
its positive and negative sided-restrictions to $\Sigma(t)$, denoted respectively by $f^{+}$and $f^{-}$, as $f^{+}(\boldsymbol{x}) \stackrel{\text { def }}{=} \lim _{\xi \rightarrow 0^{+}} f\left(\boldsymbol{x}+\xi \boldsymbol{n}^{+}\right), f^{-}(\boldsymbol{x}) \stackrel{\text { def }}{=} \lim _{\xi \rightarrow 0^{+}} f\left(\boldsymbol{x}+\xi \boldsymbol{n}^{-}\right)$for all $\boldsymbol{x} \in \Sigma(t)$. We shall also make use of the following jump operators across the interface $\Sigma(t)$ : $\llbracket f \rrbracket \stackrel{\text { def }}{=} f^{+}-f^{-}, \quad \llbracket f \boldsymbol{n} \rrbracket \stackrel{\text { def }}{=} f^{+} \boldsymbol{n}^{+}+f^{-} \boldsymbol{n}^{-}$.

The considered nonlinear coupled problem reads as follows: find the fluid velocity and pressure $\boldsymbol{u}: \Omega \times \mathbb{R}^{+} \rightarrow \mathbb{R}^{d}, p: \Omega \times \mathbb{R}^{+} \rightarrow \mathbb{R}$ and the solid displacement and velocity $\boldsymbol{d}: \Sigma \times \mathbb{R}^{+} \rightarrow \mathbb{R}^{d}, \dot{\boldsymbol{d}}: \Sigma \times \mathbb{R}^{+} \rightarrow \mathbb{R}^{d}$ such that

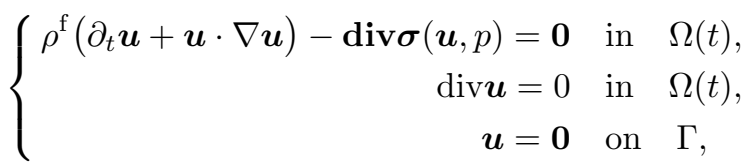

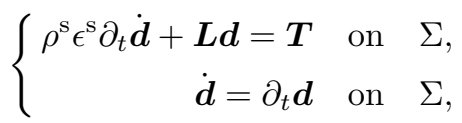

$$
\left\{\begin{array}{l}
\phi=\boldsymbol{I}_{\Sigma}+\boldsymbol{d}, \quad \Sigma(t)=\boldsymbol{\phi}(\Sigma, t), \quad \Omega(t)=\Omega \backslash \Sigma(t), \\
\boldsymbol{u}=\dot{\boldsymbol{d}} \circ \boldsymbol{\phi}_{t}^{-1} \quad \text { on } \quad \Sigma(t), \\
\int_{\Sigma} \boldsymbol{T} \cdot \boldsymbol{w}=-\int_{\Sigma(t)} \llbracket \boldsymbol{\sigma}(\boldsymbol{u}, p) \boldsymbol{n} \rrbracket \cdot \boldsymbol{w} \circ \boldsymbol{\phi}_{t}^{-1}
\end{array}\right.
$$

for all smooth test functions $\boldsymbol{w}: \Sigma \rightarrow \mathbb{R}^{d}$. The above coupled system has to be complemented with appropriate initial conditions $\boldsymbol{u}(0)=\boldsymbol{u}_{0}, \boldsymbol{d}(0)=\boldsymbol{d}_{0}$ and $\dot{\boldsymbol{d}}(0)=\dot{\boldsymbol{d}}_{0}$. Here, $\rho^{\mathrm{f}}$ and $\rho^{\mathrm{s}}$ respectively denote the fluid and solid densities, $\epsilon^{\mathrm{s}}$ the thickness of the solid, and the fluid Cauchy stress tensor is given by

$$
\boldsymbol{\sigma}(\boldsymbol{u}, p) \stackrel{\text { def }}{=} 2 \mu \boldsymbol{\epsilon}(\boldsymbol{u})-p \boldsymbol{I}, \quad \boldsymbol{\epsilon}(\boldsymbol{u}) \stackrel{\text { def }}{=} \frac{1}{2}\left(\boldsymbol{\nabla} \boldsymbol{u}+\boldsymbol{\nabla} \boldsymbol{u}^{\mathrm{T}}\right),
$$

where $\mu$ denotes the fluid dynamic viscosity. The symbol $\boldsymbol{T}$ is the force applied to the structure whereas the symbol $\boldsymbol{L}$ represents an abstract surface differential operator that describes the (possibly nonlinear) elastic behavior of the structure. The three relations in (2.3) enforce, respectively, the geometric, kinematic, and dynamic interface coupling conditions. Note that the midsurface of the solid is identified with the fluid-structure interface, by neglecting all the solid thickness effects in the interface coupling.

3. Weak form with Lagrange multipliers and spatial discretization. In what follows, the closed subspaces $H_{\Gamma}^{1}(\omega)$, of functions in $H^{1}(\omega)$ with zero trace on $\Gamma$, and $L_{0}^{2}(\omega)$, of functions in $L^{2}(\omega)$ with zero mean in $\omega$, will be used. The scalar product in $L^{2}(\omega)$ is denoted by $(\cdot, \cdot)_{\omega}$, and we set $(\cdot, \cdot) \stackrel{\text { def }}{=}(\cdot, \cdot)_{\Omega}$.

We consider $\boldsymbol{V} \stackrel{\text { def }}{=}\left[H_{\Gamma}^{1}(\Omega)\right]^{d}$ and $Q \stackrel{\text { def }}{=} L_{0}^{2}(\Omega)$ as the fluid velocity and pressure functional spaces, respectively. The standard Navier-Stokes trilinear form

$$
a^{\mathrm{f}}(\boldsymbol{z} ;(\boldsymbol{u}, p),(\boldsymbol{v}, q)) \stackrel{\text { def }}{=} \rho^{\mathrm{f}}(\boldsymbol{z} \cdot \boldsymbol{\nabla} \boldsymbol{u}, \boldsymbol{v})+2 \mu(\boldsymbol{\epsilon}(\boldsymbol{u}), \boldsymbol{\epsilon}(\boldsymbol{v}))-(p, \operatorname{div} \boldsymbol{v})+(q, \operatorname{div} \boldsymbol{u})
$$

will also be used. The space of solid admissible displacements is denoted by $\boldsymbol{W} \subset$ $\left[H^{1}(\Sigma)\right]^{d}$. The weak form of the solid elastic operator $\boldsymbol{L}$ will be represented by an application $a^{\mathrm{s}}: \boldsymbol{W} \times \boldsymbol{W} \rightarrow \mathbb{R}$, which is assumed to be linear only with respect to the second argument. 
In the spirit of [11] (see also $[18,4]$ ), we introduce a space of Lagrange multipliers $\boldsymbol{\Lambda}$ and a continuous bilinear form $b: \boldsymbol{\Lambda} \times\left[H^{\frac{1}{2}}(\Sigma)\right]^{d} \rightarrow \mathbb{R}$ such that $b(\boldsymbol{\mu}, \boldsymbol{z})=0$ for all $\boldsymbol{\mu} \in \boldsymbol{\Lambda}$ implies $\boldsymbol{z}=\mathbf{0}$ on $\Sigma$. As an example, we can take $\boldsymbol{\Lambda}=\left(\left[H^{\frac{1}{2}}(\Sigma)\right]^{d}\right)^{\prime}$ and $b(\boldsymbol{\mu}, \boldsymbol{z})=\langle\boldsymbol{\mu}, \boldsymbol{z}\rangle$, where $\langle\cdot, \cdot\rangle$ represents the duality pairing between $\left(\left[H^{\frac{1}{2}}(\Sigma)\right]^{d}\right)^{\prime}$ and $\left[H^{\frac{1}{2}}(\Sigma)\right]^{d}$ (see, e.g., $\left.[11,42]\right)$.

The weak form of the linear coupled problem (2.1)-(2.3) reads therefore as follows: for $t>0$, find $(\boldsymbol{u}, p, \boldsymbol{d}, \boldsymbol{\lambda}) \in \boldsymbol{V} \times Q \times \boldsymbol{W} \times \boldsymbol{\Lambda}$, with $\dot{\boldsymbol{d}}=\partial_{t} \boldsymbol{d}$ such that the geometric compatibility $(2.3)_{1}$ holds and

$$
\begin{aligned}
\rho^{\mathrm{f}}\left(\partial_{t} \boldsymbol{u}, \boldsymbol{v}\right)+a^{\mathrm{f}}(\boldsymbol{u} ;(\boldsymbol{u}, p),(\boldsymbol{v}, q)) & +\rho^{\mathrm{s}} \epsilon^{\mathrm{s}}\left(\partial_{t} \dot{\boldsymbol{d}}, \boldsymbol{w}\right)_{\Sigma}+a^{\mathrm{s}}(\boldsymbol{d}, \boldsymbol{w}) \\
& +b(\boldsymbol{\lambda}, \boldsymbol{v} \circ \boldsymbol{\phi}-\boldsymbol{w})-b(\boldsymbol{\mu}, \boldsymbol{u} \circ \boldsymbol{\phi}-\dot{\boldsymbol{d}})=0
\end{aligned}
$$

for all $(\boldsymbol{v}, q, \boldsymbol{w}, \boldsymbol{\mu}) \in \boldsymbol{V} \times Q \times \boldsymbol{W} \times \boldsymbol{\Lambda}$.

We now consider a family $\left\{\mathcal{T}_{h}^{\mathrm{f}}\right\}_{0<h<1}$ of triangulations of $\Omega$. The mesh $\mathcal{T}_{h}^{\mathrm{f}}$ is fitted to the exterior boundary $\Gamma$ but, in general, not to $\Sigma$. For the solid, we consider a family $\left\{\mathcal{T}_{h}^{\mathrm{s}}\right\}_{0<h<1}$ of triangulations of $\Sigma$. We introduce the following standard spaces of continuous piecewise affine functions:

$$
\begin{array}{ll}
X_{h}^{\mathrm{f}} \stackrel{\text { def }}{=}\left\{v_{h} \in C^{0}(\bar{\Omega}) / v_{h \mid K} \in \mathbb{P}_{1}(K)\right. & \left.\forall K \in \mathcal{T}_{h}^{\mathrm{f}}\right\}, \\
X_{h}^{\text {s def }} \stackrel{\text { def }}{=}\left\{v_{h} \in C^{0}(\bar{\Sigma}) / v_{h \mid K} \in \mathbb{P}_{1}(K) \quad \forall K \in \mathcal{T}_{h}^{\mathrm{s}}\right\} .
\end{array}
$$

For the approximations of the fluid velocity and pressure, we will consider the spaces

$$
\boldsymbol{V}_{h} \stackrel{\text { def }}{=}\left[X_{h}^{\mathrm{f}}\right]^{d} \cap \boldsymbol{V}, \quad Q_{h} \stackrel{\text { def }}{=} X_{h}^{\mathrm{f}} \cap Q,
$$

respectively. Furthermore, we consider the following discrete counterpart of (3.1):

$$
\begin{aligned}
a_{h}^{\mathrm{f}}\left(\boldsymbol{z}_{h} ;\left(\boldsymbol{u}_{h}, p_{h}\right),\left(\boldsymbol{v}_{h}, q_{h}\right)\right) \stackrel{\text { def }}{=} & a^{\mathrm{f}}\left(\boldsymbol{z}_{h} ;\left(\boldsymbol{u}_{h}, p_{h}\right),\left(\boldsymbol{v}_{h}, q_{h}\right)\right)+\frac{\rho^{\mathrm{f}}}{2}\left(\left(\operatorname{div} \boldsymbol{z}_{h}\right) \boldsymbol{u}_{h}, \boldsymbol{v}_{h}\right) \\
& +s_{h}\left(\boldsymbol{z}_{h} ; \boldsymbol{u}_{h}, \boldsymbol{v}_{h}\right),
\end{aligned}
$$

where the form $s_{h}$ corresponds to the SUPG/PSPG and grad-div stabilizations given by (see, e.g., $[47,34])$ :

$$
\begin{aligned}
s_{h}\left(\boldsymbol{z}_{h} ; \boldsymbol{u}_{h}, \boldsymbol{v}_{h}\right) \stackrel{\text { def }}{=} & \sum_{K \in \mathcal{T}_{h}^{\mathrm{f}}} \int_{K} \frac{\lambda_{\mathrm{C}} h^{2}}{\delta_{h}} \operatorname{div} \boldsymbol{u}_{h} \operatorname{div} \boldsymbol{v}_{h} \\
& +\sum_{K \in \mathcal{T}_{h}^{\mathrm{f}}} \int_{K} \delta_{h}\left(\rho^{\mathrm{f}}\left(\boldsymbol{z}_{h} \cdot \nabla\right) \boldsymbol{u}_{h}+\nabla p_{h}\right) \cdot\left(\rho^{\mathrm{f}}\left(\boldsymbol{z}_{h} \cdot \nabla\right) \boldsymbol{v}_{h}+\nabla q_{h}\right), \\
\delta_{h} \stackrel{\text { def }}{=} & \lambda_{\mathrm{M}}\left(\rho^{\mathrm{f}} \sqrt{\frac{4}{\tau^{2}}+\frac{16 \mu^{2}}{h^{4}\left(\rho^{\mathrm{f}}\right)^{2}}+\frac{4\left|\boldsymbol{z}_{h}\right|^{2}}{h^{2}}}\right)^{-1},
\end{aligned}
$$

with $\lambda_{\mathrm{M}}>0$ and $\lambda_{\mathrm{C}} \geq 0$ user-defined parameters.

In order to overcome the artificial interfacial mass losses induced by the continuous nature of the pressure approximations considered in (3.4), we will consider (notably when dealing with enclosed fluid domains) the approach proposed in [34] for an immersogeometric method, which consists in boosting the grad-div stabilization while reducing the SUPG/PSPG stabilization near the interface by taking (see also $[16,12])$ : 


$$
\lambda_{\mathrm{C}}=1 \quad \text { in } \quad \Omega, \quad \lambda_{\mathrm{M}}=\left\{\begin{array}{rll}
1 & \text { in } & \Omega \backslash \omega_{h}^{n} \\
\varepsilon_{\mathrm{M}} & \text { in } & \omega_{h}^{n}
\end{array}\right.
$$

where $0<\varepsilon_{\mathrm{M}} \ll 1$ is a user-defined (dimensionless) parameter and $\omega_{h}^{n}$ a neighborhood of the interface $\Sigma_{h}^{n}$ (typically two layers of fluid elements on each of its side). The motivation of the first choice is that it improves local mass conservation while the second reduces the impact of the local residual inconsistencies near the interface.

The solid displacement and velocity are approximated in $\boldsymbol{W}_{h} \stackrel{\text { def }}{=}\left[X_{h}^{\mathrm{s}}\right]^{d} \cap \boldsymbol{W}$. For the approximation of the Lagrange multiplier, we consider the following nonconforming approximation space (see, e.g., $[8,18,30,19])$ :

$$
\boldsymbol{\Lambda}_{h}=\left\{\boldsymbol{\mu}_{h}=\sum_{i=1}^{N_{h}^{\mathrm{s}}} \boldsymbol{\mu}_{i} \delta_{\boldsymbol{x}_{i}^{\mathrm{s}}} / \boldsymbol{\mu}_{i} \in \mathbb{R}^{d}, \quad i=1, \ldots, N_{h}^{\mathrm{s}}\right\},
$$

where $\left\{\boldsymbol{x}_{i}^{\mathrm{s}}\right\}_{i=1}^{N_{h}^{\mathrm{s}}}$ denotes the points of the triangulation $\mathcal{T}_{h}^{\mathrm{s}}$ and $\delta_{\boldsymbol{x}_{i}^{\mathrm{s}}}$ stands for the Dirac's measure at point $\boldsymbol{x}_{i}^{\mathrm{s}}$. For alternative approximation spaces, the reader is referred to $[4,2,11]$, for instance. Due to the nonconforming nature of the approximation (3.7), we introduce the discrete bilinear form $b_{h}: \boldsymbol{\Lambda}_{h} \times\left[C^{0}(\Sigma)\right]^{d} \rightarrow \mathbb{R}$, defined by

$$
b_{h}\left(\boldsymbol{\mu}_{h}, \boldsymbol{z}\right) \stackrel{\text { def }}{=} \sum_{i=1}^{N_{h}^{\mathrm{s}}} \boldsymbol{\mu}_{i} \cdot \boldsymbol{z}\left(\boldsymbol{x}_{i}^{\mathrm{s}}\right)
$$

for all $\left(\boldsymbol{\mu}_{h}, \boldsymbol{z}\right) \in \boldsymbol{\Lambda}_{h} \times\left[C^{0}(\Sigma)\right]^{d}$. This amounts to enforce the kinematic constraint $(2.3)_{2}$ as in a collocation method (see, e.g., $[8,30]$ ). The spatial semidiscrete approximation of (3.2) reads therefore as follows: for $t>0$, find $\left(\boldsymbol{u}_{h}, p_{h}, \boldsymbol{d}_{h}, \boldsymbol{\lambda}_{h}\right) \in$ $\boldsymbol{V}_{h} \times Q_{h} \times \boldsymbol{W}_{h} \times \boldsymbol{\Lambda}_{h}$, with $\dot{\boldsymbol{d}}_{h}=\partial_{t} \boldsymbol{d}_{h}, \boldsymbol{\phi}_{h}=\boldsymbol{I}_{\Sigma}+\boldsymbol{d}_{h}$ and such that

$$
\begin{aligned}
\rho^{\mathrm{f}}\left(\partial_{t} \boldsymbol{u}_{h}, \boldsymbol{v}_{h}\right) & +a_{h}^{\mathrm{f}}\left(\boldsymbol{u}_{h} ;\left(\boldsymbol{u}_{h}, p_{h}\right),\left(\boldsymbol{v}_{h}, q_{h}\right)\right)+b_{h}\left(\boldsymbol{\lambda}_{h}, \boldsymbol{v}_{h} \circ \boldsymbol{\phi}_{h}-\boldsymbol{w}_{h}\right) \\
& +\rho^{\mathrm{s}} \epsilon^{\mathrm{s}}\left(\partial_{t} \dot{\boldsymbol{d}}_{h}, \boldsymbol{w}_{h}\right)_{\Sigma}+a^{\mathrm{s}}\left(\boldsymbol{d}_{h}, \boldsymbol{w}_{h}\right)-b_{h}\left(\boldsymbol{\mu}_{h}, \boldsymbol{u}_{h} \circ \boldsymbol{\phi}_{h}-\dot{\boldsymbol{d}}_{h}\right)=0
\end{aligned}
$$

for all $\left(\boldsymbol{v}_{h}, q_{h}, \boldsymbol{w}_{h}, \boldsymbol{\mu}_{h}\right) \in \boldsymbol{V}_{h} \times Q_{h} \times \boldsymbol{W}_{h} \times \boldsymbol{\Lambda}_{h}$.

4. Time-discretization: Coupling schemes. This section is devoted to the discretization in time of (3.9). In what follows, the parameter $\tau>0$ stands for the time-step length and $t_{n} \stackrel{\text { def }}{=} n \tau$, for $n \in \mathbb{N}$. For a given time-dependent field $x(t)$, the symbol $x^{n}$ denotes an approximation of $x\left(t_{n}\right)$ and $\partial_{\tau} x^{n} \stackrel{\text { def }}{=}\left(x^{n}-x^{n-1}\right) / \tau$, the firstorder backward difference. For simplicity, we consider a first-order time-discretization of the bulk terms in the fluid and in the solid.

We first introduce the strongly coupled scheme reported in Algorithm 4.1 (see, e.g., $[11,9]$ ). The method implicitly treats the kinematic-dynamic coupling through the Lagrange multiplier, but the geometric coupling is treated in an explicit fashion. This yields unconditional stability but at the price of solving the coupled system (4.3) below at each time-step, which can be costly and cumbersome (e.g., when the fluid and the solid are solved in separate codes).

Owing to (3.8), the discrete kinematic constraint in (4.3) writes

$$
\boldsymbol{u}_{h}^{n} \circ \phi_{h}^{n}\left(\boldsymbol{x}_{i}^{\mathrm{s}}\right)-\dot{\boldsymbol{d}}_{h}^{n}\left(\boldsymbol{x}_{i}^{\mathrm{s}}\right)=0 \quad \forall i=1, \ldots, N_{h}^{\mathrm{s}} .
$$




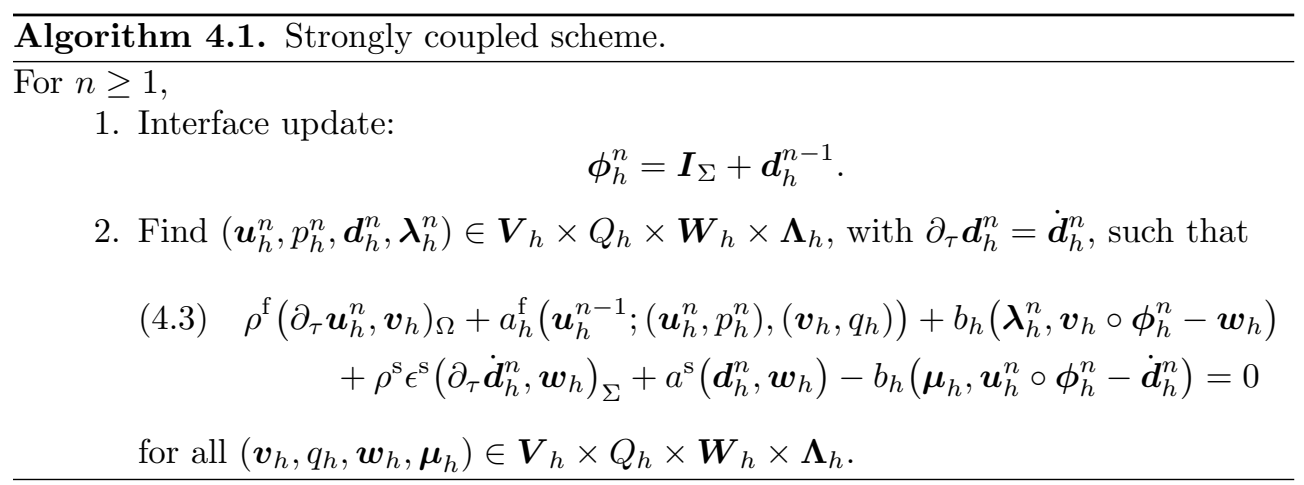

This is also equivalent to consider in (4.3) (and in (3.9)) the conforming space of Lagrange multipliers $\boldsymbol{\Lambda}_{h}=\left[X_{h}^{\mathrm{s}}\right]^{d}$ and the discrete bilinear form $b_{h}\left(\boldsymbol{\mu}_{h}, \boldsymbol{z}\right)=\left(\boldsymbol{\mu}_{h}, \boldsymbol{z}\right)_{\Sigma, h}$. The symbol $(\cdot, \cdot)_{\Sigma, h}$ denotes the lumped-mass approximation of the $L^{2}$-inner product $(\cdot, \cdot)_{\Sigma}$, namely, the surface integral over $\Sigma$ is approximated using nodal quadrature. Note that (4.1) avoids the need for the evaluation of interface integrals with quantities defined on unfitted meshes. Actually, only localization of the solid nodes within the fluid mesh is required. Little is known however on the discrete inf-sup conditions guaranteeing the existence, uniqueness, and convergence of the approximation provided by (4.3), for these choices of the Lagrange multipliers spaces.

Remark 4.1. In this regard, we are only aware of two theoretical results. The first concerns the convergence analysis reported in [19, section 3.2] for the primal variable of a saddle-point problem involving the Poisson equation, provided that the local size of the solid mesh is of the same order as the local size of the fluid mesh. More recently, a complete analysis is given in [11, section 5] for the choice $\boldsymbol{\Lambda}_{h}=\left[X_{h}^{\mathrm{s}}\right]^{d}$ and $b_{h}\left(\boldsymbol{\mu}_{h}, \boldsymbol{z}\right)=b\left(\boldsymbol{\mu}_{h}, \boldsymbol{z}\right)$ (i.e., without quadrature approximation of the interface integral), under the assumption that the fluid mesh is sufficiently refined with respect to the solid mesh.

In other to avoid the lack of inf-sup stability result for (4.3), we follow the penalty strategy considered in [18] for the computer implementation of Algorithm 4.1, which consists in relaxing (4.1) to

$$
\boldsymbol{u}_{h}^{n} \circ \phi_{h}^{n}\left(\boldsymbol{x}_{i}^{\mathbf{s}}\right)-\dot{\boldsymbol{d}}_{h}^{n}\left(\boldsymbol{x}_{i}^{\mathrm{s}}\right)=\varepsilon \boldsymbol{\lambda}_{i}^{n} \quad \forall i=1, \ldots, N_{h}^{\mathrm{s}},
$$

where $\varepsilon>0$ is a small (nondimensionless) parameter. This enables the elimination of the Lagrange multipliers, with the convenient property of preserving the sparse pattern of the matrix of the fluid problem. The fundamental drawbacks of this approach lie in the choice of the parameter $\varepsilon$ (which needs be tuned depending on the mesh size; see [12]) and in the ill-conditioning issues induced by the resulting penalty term in the fluid momentum equation.

We now consider the alternative numerical method reported in Algorithm 4.2 that is not strongly coupled and, hence, less computationally demanding than Algorithm 4.1. This scheme, introduced in [2] for a different choice of $\boldsymbol{\Lambda}_{h}$, extends the ideas of $[20,1]$ to the unfitted mesh formulation (3.9). Basically, this scheme treats implicitly the coupling of the fluid with the solid inertia and explicitly the coupling with the solid elastic effects. The former guarantees stability (by avoiding the explicit treatment of the added-mass) while the latter reduces the computational complexity with respect to Algorithm 4.1. 


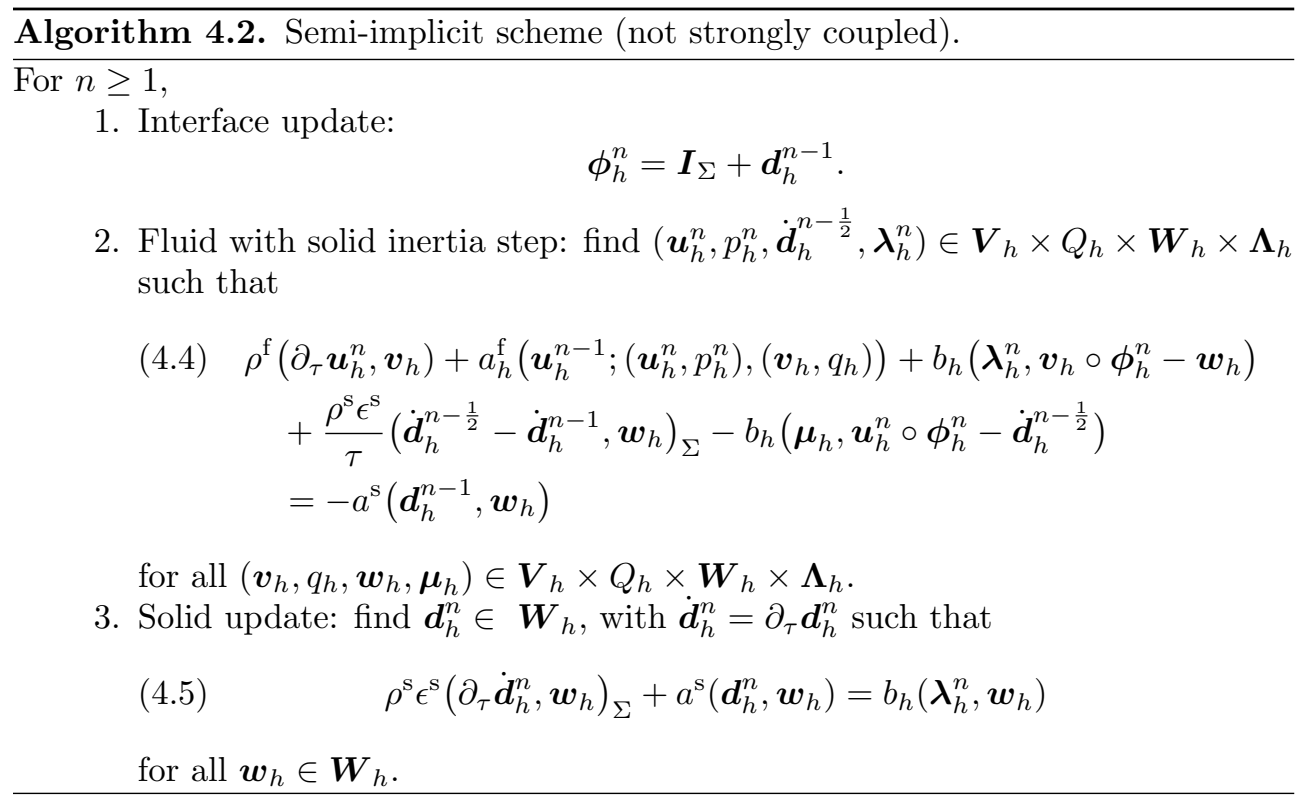

Remark 4.2. Alternative extrapolations (e.g., zeroth or second order) could be considered for the last term of (4.4), as reported in [20,1,2]. Nevertheless, in the present work, we limit the discussion to first-order extrapolation since it guarantees both unconditional stability (Theorem 4.9) and first-order time accuracy.

Note that Algorithm 4.2 uncouples the computation of the fluid and solid unknowns $\left(\boldsymbol{u}_{h}^{n}, p_{h}^{n}, \boldsymbol{\lambda}_{h}^{n}\right)$ and $\left(\dot{\boldsymbol{d}}_{h}^{n}, \boldsymbol{d}_{h}^{n}\right)$. The price to pay for this splitting is the introduction of a new unknown in step (4.4), the so-called intermediate solid velocity $\dot{\boldsymbol{d}}_{h}^{n-\frac{1}{2}}$. Similar difficulties arise in the semi-implicit scheme reported in [1, Algorithm 6] for a Nitsche-XFEM unfitted mesh method (Lagrange multipliers free).

4.1. A new loosely coupled scheme. The first fundamental idea of the present paper is that, if we choose $\boldsymbol{\Lambda}_{h}$ as in (3.7), both the intermediate velocity $\dot{\boldsymbol{d}}_{h}^{n-\frac{1}{2}}$ and the Lagrange multiplier $\boldsymbol{\lambda}_{h}^{n}$ can be eliminated in terms of the standard fluid unknown $\left(\boldsymbol{u}_{h}^{n}, p_{h}^{n}\right)$. To this purpose, we introduce the fluid-to-solid Lagrange interpolation operator

$$
\boldsymbol{B}_{h}:\left[C^{0}(\Sigma)\right]^{d} \rightarrow \boldsymbol{W}_{h},
$$

and we state the following result.

Lemma 4.3. Let the discrete space $\boldsymbol{\Lambda}_{h}$ be given by (3.7). We have

$$
b_{h}\left(\boldsymbol{\mu}_{h}, \boldsymbol{v}_{h} \circ \boldsymbol{\phi}_{h}^{n}\right)=b_{h}\left(\boldsymbol{\mu}_{h}, \boldsymbol{B}_{h}\left(\boldsymbol{v}_{h} \circ \boldsymbol{\phi}_{h}^{n}\right)\right) \quad \forall \boldsymbol{v}_{h} \in \boldsymbol{V}_{h} .
$$

Furthermore, the relation

$$
b_{h}\left(\boldsymbol{\mu}_{h}, \boldsymbol{v}_{h} \circ \phi_{h}^{n}-\boldsymbol{w}_{h}\right)=0 \quad \forall \boldsymbol{\mu}_{h} \in \boldsymbol{\Lambda}_{h}
$$

is equivalent to

$$
\boldsymbol{w}_{h}=\boldsymbol{B}_{h}\left(\boldsymbol{v}_{h} \circ \phi_{h}^{n}\right) .
$$


Proof. From (3.8), we have

$$
\begin{aligned}
b_{h}\left(\boldsymbol{\mu}_{h}, \boldsymbol{v}_{h} \circ \boldsymbol{\phi}_{h}^{n}\right)=\sum_{i=1}^{N_{h}^{\mathrm{s}}} \boldsymbol{\mu}_{i} \cdot \boldsymbol{v}_{h}\left(\phi_{h}^{n}\left(\boldsymbol{x}_{i}^{\mathrm{s}}\right)\right) & =\sum_{i=1}^{N_{h}^{\mathrm{s}}} \boldsymbol{\mu}_{i} \cdot \boldsymbol{B}_{h}\left(\boldsymbol{v}_{h} \circ \boldsymbol{\phi}_{h}^{n}\right)\left(\boldsymbol{x}_{i}^{\mathrm{s}}\right) \\
& =b_{h}\left(\boldsymbol{\mu}_{h}, \boldsymbol{B}_{h}\left(\boldsymbol{v}_{h} \circ \boldsymbol{\phi}_{h}^{n}\right)\right) .
\end{aligned}
$$

On the other hand, owing to (4.7), we get

$$
\sum_{i=1}^{N_{h}^{\mathrm{s}}} \boldsymbol{\mu}_{i} \cdot\left(\boldsymbol{B}_{h}\left(\boldsymbol{v}_{h} \circ \boldsymbol{\phi}_{h}^{n}\right)\left(\boldsymbol{x}_{i}^{\mathbf{s}}\right)-\boldsymbol{w}_{h}\left(\boldsymbol{x}_{i}^{\mathbf{s}}\right)\right)=0
$$

for all $\boldsymbol{\mu}_{i} \in \mathbb{R}^{d}$, or, equivalently,

$$
\boldsymbol{B}_{h}\left(\boldsymbol{v}_{h} \circ \phi_{h}^{n}\right)\left(\boldsymbol{x}_{i}^{\mathrm{s}}\right)=\boldsymbol{w}_{h}\left(\boldsymbol{x}_{i}^{\mathrm{s}}\right)
$$

for $i=1, \ldots, N_{h}^{\mathrm{s}}$, which yields (4.8) and completes the proof.

The next result shows that the coupled system (4.4) can be formulated exclusively in terms of a pure fluid problem without additional unknowns.

LEMma 4.4. For $n \geq 1$, let $\left(\boldsymbol{u}_{h}^{n}, p_{h}^{n}, \dot{\boldsymbol{d}}_{h}^{n-\frac{1}{2}}, \boldsymbol{\lambda}_{h}^{n}\right) \in \boldsymbol{V}_{h} \times Q_{h} \times \boldsymbol{W}_{h} \times \boldsymbol{\Lambda}_{h}$ be solution of (4.4), then we have:

- $\dot{\boldsymbol{d}}_{h}^{n-\frac{1}{2}}=\boldsymbol{B}_{h}\left(\boldsymbol{u}_{h}^{n} \circ \boldsymbol{\phi}_{h}^{n}\right)$;

- $\left(\boldsymbol{u}_{h}^{n}, p_{h}^{n}\right) \in \boldsymbol{V}_{h} \times Q_{h}$ satisfies

$$
\begin{aligned}
& \rho^{\mathrm{f}}\left(\partial_{\tau} \boldsymbol{u}_{h}^{n}, \boldsymbol{v}_{h}\right)+a_{h}^{\mathrm{f}}\left(\boldsymbol{u}_{h}^{n-1} ;\left(\boldsymbol{u}_{h}^{n}, p_{h}^{n}\right),\left(\boldsymbol{v}_{h}, q_{h}\right)\right) \\
& \quad+\frac{\rho^{\mathrm{s}} \epsilon^{\mathrm{s}}}{\tau}\left(\boldsymbol{B}_{h}\left(\boldsymbol{u}_{h}^{n} \circ \boldsymbol{\phi}_{h}^{n}\right), \boldsymbol{B}_{h}\left(\boldsymbol{v}_{h} \circ \boldsymbol{\phi}_{h}^{n}\right)\right)_{\Sigma} \\
& =\frac{\rho^{\mathrm{s}} \epsilon^{\mathrm{s}}}{\tau}\left(\dot{\boldsymbol{d}}_{h}^{n-1}, \boldsymbol{B}_{h}\left(\boldsymbol{v}_{h} \circ \boldsymbol{\phi}_{h}^{n}\right)\right)_{\Sigma}-a^{\mathrm{s}}\left(\boldsymbol{d}_{h}^{n-1}, \boldsymbol{B}_{h}\left(\boldsymbol{v}_{h} \circ \boldsymbol{\phi}_{h}^{n}\right)\right)
\end{aligned}
$$

for all $\left(\boldsymbol{v}_{h}, q_{h}\right) \in \boldsymbol{V}_{h} \times Q_{h}$

- $\boldsymbol{\lambda}_{h}^{n} \in \boldsymbol{\Lambda}_{h}$ satisfies

$$
b\left(\boldsymbol{\lambda}_{h}^{n}, \boldsymbol{w}_{h}\right)=\frac{\rho^{\mathrm{s}} \epsilon^{\mathrm{s}}}{\tau}\left(\boldsymbol{B}_{h}\left(\boldsymbol{u}_{h}^{n} \circ \boldsymbol{\phi}_{h}^{n}\right)-\dot{\boldsymbol{d}}_{h}^{n-1}, \boldsymbol{w}_{h}\right)_{\Sigma}+a^{\mathrm{s}}\left(\boldsymbol{d}_{h}^{n-1}, \boldsymbol{w}_{h}\right)
$$

for all $\boldsymbol{w}_{h} \in \boldsymbol{W}_{h}$.

The reciprocal also holds.

Proof. From (4.4) with $\left(\boldsymbol{v}_{h}, q_{h}, \boldsymbol{w}_{h}\right)=(\mathbf{0}, 0, \mathbf{0})$, we have

$$
b_{h}\left(\boldsymbol{\mu}_{h}, \boldsymbol{u}_{h}^{n} \circ \boldsymbol{\phi}_{h}^{n}-\dot{\boldsymbol{d}}_{h}^{n-\frac{1}{2}}\right)=0 \quad \forall \boldsymbol{\mu}_{h} \in \boldsymbol{\Lambda}_{h}
$$

and

$$
\begin{aligned}
\rho^{\mathrm{f}}\left(\partial_{\tau} \boldsymbol{u}_{h}^{n}, \boldsymbol{v}_{h}\right) & +a_{h}^{\mathrm{f}}\left(\boldsymbol{u}_{h}^{n-1} ;\left(\boldsymbol{u}_{h}^{n}, p_{h}^{n}\right),\left(\boldsymbol{v}_{h}, q_{h}\right)\right)+b_{h}\left(\boldsymbol{\lambda}_{h}^{n}, \boldsymbol{v}_{h} \circ \boldsymbol{\phi}_{h}^{n}-\boldsymbol{w}_{h}\right) \\
& +\frac{\rho^{\mathrm{s}} \epsilon^{\mathrm{s}}}{\tau}\left(\dot{\boldsymbol{d}}_{h}^{n-\frac{1}{2}}-\dot{\boldsymbol{d}}_{h}^{n-1}, \boldsymbol{w}_{h}\right)_{\Sigma}=-a^{\mathrm{s}}\left(\boldsymbol{d}_{h}^{n-1}, \boldsymbol{w}_{h}\right)
\end{aligned}
$$

for all $\left(\boldsymbol{v}_{h}, q_{h}, \boldsymbol{w}_{h}\right) \in \boldsymbol{V}_{h} \times Q_{h} \times \boldsymbol{W}_{h}$. Owing to Lemma 4.3, these relations can respectively be formulated as

$$
\boldsymbol{B}_{h}\left(\boldsymbol{u}_{h}^{n} \circ \boldsymbol{\phi}_{h}^{n}\right)=\dot{\boldsymbol{d}}_{h}^{n-\frac{1}{2}}
$$


and

$$
\begin{array}{r}
\rho^{\mathrm{f}}\left(\partial_{\tau} \boldsymbol{u}_{h}^{n}, \boldsymbol{v}_{h}\right)+a_{h}^{\mathrm{f}}\left(\boldsymbol{u}_{h}^{n-1} ;\left(\boldsymbol{u}_{h}^{n}, p_{h}^{n}\right),\left(\boldsymbol{v}_{h}, q_{h}\right)\right)+b_{h}\left(\boldsymbol{\lambda}_{h}^{n}, \boldsymbol{B}_{h}\left(\boldsymbol{v}_{h} \circ \boldsymbol{\phi}_{h}^{n}\right)-\boldsymbol{w}_{h}\right) \\
+\frac{\rho^{\mathrm{s}} \epsilon^{\mathrm{s}}}{\tau}\left(\dot{\boldsymbol{d}}_{h}^{n-\frac{1}{2}}-\dot{\boldsymbol{d}}_{h}^{n-1}, \boldsymbol{w}_{h}\right)_{\Sigma}=-a^{\mathrm{s}}\left(\boldsymbol{d}_{h}^{n-1}, \boldsymbol{w}_{h}\right)
\end{array}
$$

for all $\left(\boldsymbol{v}_{h}, q_{h}, \boldsymbol{w}_{h}\right) \in \boldsymbol{V}_{h} \times Q_{h} \times \boldsymbol{W}_{h}$. Note that the intermediate solid velocity can be eliminated via (4.11). In order to also eliminate the Lagrange multipliers, we take $\boldsymbol{w}_{h}=\boldsymbol{B}_{h}\left(\boldsymbol{v}_{h} \circ \boldsymbol{\phi}_{h}^{n}\right)$ in (4.12), which yields (4.9). Finally, the relation (4.10) simply follows from (4.11) and (4.12) with $\left(\boldsymbol{v}_{h}, q_{h}\right)=(\mathbf{0}, 0)$.

Conversely, we assume now that (4.9) and (4.10) hold. From (4.10), there follows that

$$
\begin{aligned}
b\left(\boldsymbol{\lambda}_{h}^{n}, \boldsymbol{B}_{h}\left(\boldsymbol{v}_{h} \circ \boldsymbol{\phi}_{h}^{n}\right)-\boldsymbol{w}_{h}\right)-\frac{\rho^{\mathrm{s}} \epsilon^{\mathrm{s}}}{\tau}\left(\boldsymbol{B}_{h}\left(\boldsymbol{u}_{h}^{n} \circ \boldsymbol{\phi}_{h}^{n}\right)\right. & \left.-\dot{\boldsymbol{d}}_{h}^{n-1}, \boldsymbol{B}_{h}\left(\boldsymbol{v}_{h} \circ \boldsymbol{\phi}_{h}^{n}\right)-\boldsymbol{w}_{h}\right)_{\Sigma} \\
& =a^{\mathrm{s}}\left(\boldsymbol{d}_{h}^{n-1}, \boldsymbol{B}_{h}\left(\boldsymbol{v}_{h} \circ \boldsymbol{\phi}_{h}^{n}\right)-\boldsymbol{w}_{h}\right)
\end{aligned}
$$

for all $\left(\boldsymbol{v}_{h}, \boldsymbol{w}_{h}\right) \in \boldsymbol{V}_{h} \times \boldsymbol{W}_{h}$. By adding this expression to (4.9), we get

$$
\begin{aligned}
\rho^{\mathrm{f}}\left(\partial_{\tau} \boldsymbol{u}_{h}^{n}, \boldsymbol{v}_{h}\right)+a_{h}^{\mathrm{f}}\left(\boldsymbol{u}_{h}^{n-1} ;(\right. & \left.\left.\boldsymbol{u}_{h}^{n}, p_{h}^{n}\right),\left(\boldsymbol{v}_{h}, q_{h}\right)\right)+b_{h}\left(\boldsymbol{\lambda}_{h}^{n}, \boldsymbol{B}_{h}\left(\boldsymbol{v}_{h} \circ \boldsymbol{\phi}_{h}^{n}\right)-\boldsymbol{w}_{h}\right) \\
& +\frac{\rho^{\mathrm{s}} \epsilon^{\mathrm{s}}}{\tau}\left(\boldsymbol{B}_{h}\left(\boldsymbol{u}_{h}^{n} \circ \boldsymbol{\phi}_{h}^{n}\right)-\dot{\boldsymbol{d}}_{h}^{n-1}, \boldsymbol{w}_{h}\right)_{\Sigma}=-a^{\mathrm{s}}\left(\boldsymbol{d}_{h}^{n-1}, \boldsymbol{w}_{h}\right)
\end{aligned}
$$

for all $\left(\boldsymbol{v}_{h}, q_{h}, \boldsymbol{w}_{h}\right) \in \boldsymbol{V}_{h} \times Q_{h} \times \boldsymbol{W}_{h}$. We finally retrieve (4.4) by setting $\dot{\boldsymbol{d}}_{h}^{n-\frac{1}{2}}=$ $\boldsymbol{B}_{h}\left(\boldsymbol{u}_{h}^{n} \circ \phi_{h}^{n}\right)$ and by applying Lemma 4.3. This completes the proof.

Remark 4.5. Note that (4.9) is a pure fluid problem, with a specific nonnegative bilinear term acting on the interface. It is therefore well posed. Furthermore, owing to the reciprocal part of Lemma 4.4, (4.4) admits also a unique solution.

Remark 4.6. The system (4.9) can be viewed as a fluid problem with an immersed interface condition that generalizes the Robin-base splitting reported in $[20,25,37]$ to the case of unfitted meshes. Alternative interface Robin conditions (as those considered in $[41,31,13]$ with fitted meshes) can also be generalized with the present approach.

The fundamental difficulty of (4.9) is that, in general, the interfacial term introduces nonstandard coupling terms in the fluid matrix. Even more, the stencil of the resulting matrix depends on the location of the interface at each time-step. In order to overcome these drawbacks, we propose to replace the canonic $L^{2}$-inner product $(\cdot, \cdot)_{\Sigma}$ in Algorithm 4.2 by its lumped-mass approximation $(\cdot, \cdot)_{\Sigma, h}$ (see, e.g., [48, Chapter 15]). We can then establish the following result.

LEMma 4.7. The term

$$
\left(\boldsymbol{B}_{h}\left(\boldsymbol{u}_{h}^{n} \circ \boldsymbol{\phi}_{h}^{n}\right), \boldsymbol{B}_{h}\left(\boldsymbol{v}_{h} \circ \boldsymbol{\phi}_{h}^{n}\right)\right)_{\Sigma, h}
$$

preserves the sparsity of the original fluid matrix.

Proof. Let $i, j \in \mathbb{N}$ be the indices of two fluid nodes which do not share the same edge (see Figure 2). We will show that its corresponding matrix entry in each block of (4.13) vanishes. The matrix associated with (4.13) has a diagonal block structure; for instance, in two dimensions we have

$$
\mathrm{R}^{n} \stackrel{\text { def }}{=}\left[\begin{array}{cc}
\left(\mathrm{B}^{n}\right)^{\mathrm{T}} \mathrm{MB}^{n} & 0 \\
0 & \left(\mathrm{~B}^{n}\right)^{\mathrm{T}} \mathrm{MB}^{n}
\end{array}\right],
$$


where $\mathrm{M} \in \mathbb{R}^{N_{h}^{\mathrm{s}} \times N_{h}^{\mathrm{s}}}$ denotes the (scalar) lumped-mass matrix of the solid and $\mathrm{B}^{n} \in$ $\mathbb{R}^{N_{h}^{\mathrm{s}} \times N_{h}^{\mathrm{f}}}$ the (scalar) Lagrange interpolation matrix from the fluid mesh to the solid mesh of the current configuration $\phi_{h}^{n}(\Sigma)$. Therefore, it suffices to discuss only the diagonal blocks of $\mathrm{R}^{n}$.

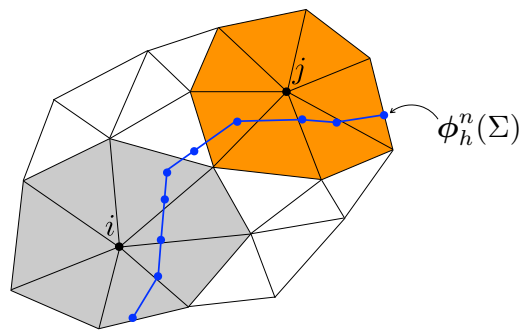

FIG. 2. The support of two fluid shape functions (in gray and in orange) intersected by the interface $\phi_{h}^{n}(\Sigma)$, where $i$ and $j$ do not share the same edge.

Since the lumped-mass matrix is diagonal, we have $\mathrm{M}_{l k}=\alpha_{l} \delta_{l k}$, with $\alpha_{l} \in \mathbb{R}$ and $\delta_{l k}$ standing for the Kronecker delta. Let $\mathrm{e}_{i}, \mathrm{e}_{j}$ be the canonical basis vectors of $\mathbb{R}^{N^{\mathrm{f}}}$ associated with the nodes $i, j$. We have

$$
\begin{aligned}
\left(\left(\mathrm{B}^{n}\right)^{\mathrm{T}} \mathrm{MB}^{n}\right)_{i j} & =\left(\mathrm{B}^{n} \mathrm{e}_{i}\right)^{\mathrm{T}} \mathrm{MB}^{n} \mathrm{e}_{j} \\
& =\sum_{l}\left(\sum_{k} \mathrm{M}_{l k}\left(\mathrm{~B}^{n} \mathrm{e}_{j}\right)_{k}\right)\left(\mathrm{B}^{n} \mathrm{e}_{i}\right)_{l}=\sum_{l} \alpha_{l}\left(\mathrm{~B}^{n} \mathrm{e}_{j}\right)_{l}\left(\mathrm{~B}^{n} \mathrm{e}_{i}\right)_{l}=0 .
\end{aligned}
$$

The last equality follows from the fact that, since the supports of the fluid nodes $i$ and $j$ do not intersect (see Figure 2), the vectors $\mathrm{B}^{n} \mathrm{e}_{j}$ and $\mathrm{B}^{n} \mathrm{e}_{i}$ do not have any common nonzero entry. This completes the proof.

Owing to the results of Lemmas 4.4 and 4.7, we introduce the following new solution procedure.

For $n \geq 1$,

1. Interface update: $\boldsymbol{\phi}_{h}^{n}=\boldsymbol{I}_{\boldsymbol{\Sigma}}+\boldsymbol{d}_{h}^{n-1}$.

2. Find $\left(\boldsymbol{u}_{h}^{n}, p_{h}^{n}\right) \in \boldsymbol{V}_{h} \times Q_{h}$ such that

$$
\begin{aligned}
\rho^{\mathrm{f}} & \left(\partial_{\tau} \boldsymbol{u}_{h}^{n}, \boldsymbol{v}_{h}\right)_{\Omega}+a_{h}^{\mathrm{f}}\left(\boldsymbol{u}_{h}^{n-1} ;\left(\boldsymbol{u}_{h}^{n}, p_{h}^{n}\right),\left(\boldsymbol{v}_{h}, q_{h}\right)\right) \\
& +\frac{\rho^{\mathrm{s}} \epsilon^{\mathrm{s}}}{\tau}\left(\boldsymbol{B}_{h}\left(\boldsymbol{u}_{h}^{n} \circ \boldsymbol{\phi}_{h}^{n}\right), \boldsymbol{B}_{h}\left(\boldsymbol{v}_{h} \circ \boldsymbol{\phi}_{h}^{n}\right)\right)_{\Sigma, h} \\
= & \frac{\rho^{\mathrm{s}} \epsilon^{\mathrm{s}}}{\tau}\left(\dot{\boldsymbol{d}}_{h}^{n-1}, \boldsymbol{B}_{h}\left(\boldsymbol{v}_{h} \circ \boldsymbol{\phi}_{h}^{n}\right)\right)_{\Sigma, h}-a^{\mathrm{s}}\left(\boldsymbol{d}_{h}^{n-1}, \boldsymbol{B}_{h}\left(\boldsymbol{v}_{h} \circ \boldsymbol{\phi}_{h}^{n}\right)\right)
\end{aligned}
$$

for all $\left(\boldsymbol{v}_{h}, q_{h}\right) \in \boldsymbol{V}_{h} \times Q_{h}$.

3. Find $\boldsymbol{\lambda}_{h}^{n} \in \boldsymbol{\Lambda}_{h}$ such that

$$
b_{h}\left(\boldsymbol{\lambda}_{h}^{n}, \boldsymbol{w}_{h}\right)=\frac{\rho^{\mathrm{s}} \epsilon^{\mathrm{s}}}{\tau}\left(\boldsymbol{B}_{h}\left(\boldsymbol{u}_{h}^{n} \circ \boldsymbol{\phi}_{h}^{n}\right)-\dot{\boldsymbol{d}}_{h}^{n-1}, \boldsymbol{w}_{h}\right)_{\Sigma}+a^{\mathrm{s}}\left(\boldsymbol{d}_{h}^{n-1}, \boldsymbol{w}_{h}\right)
$$

for all $\boldsymbol{w}_{h} \in \boldsymbol{W}_{h}$.

4. Find $\boldsymbol{d}_{h}^{n} \in \boldsymbol{W}_{h}$, with $\dot{\boldsymbol{d}}_{h}^{n}=\partial_{\tau} \boldsymbol{d}_{h}^{n}$, such that

$$
\rho^{\mathrm{s}} \epsilon^{\mathrm{s}}\left(\partial_{\tau} \dot{\boldsymbol{d}}_{h}^{n}, \boldsymbol{w}_{h}\right)_{\Sigma, h}+a^{\mathrm{s}}\left(\boldsymbol{d}_{h}^{n}, \boldsymbol{w}_{h}\right)=b_{h}\left(\boldsymbol{\lambda}_{h}^{n}, \boldsymbol{w}_{h}\right)
$$

for all $\boldsymbol{w}_{h} \in \boldsymbol{W}_{h}$. 
From a practical point of view, it is worth noting that, using (4.16), the relations (4.14)-(4.15) can also be rewritten equivalently, by replacing the terms containing $\boldsymbol{d}_{h}^{n-1}$, as

$$
\begin{aligned}
\rho^{\mathrm{f}} & \left(\partial_{\tau} \boldsymbol{u}_{h}^{n}, \boldsymbol{v}_{h}\right)+a_{h}^{\mathrm{f}}\left(\boldsymbol{u}_{h}^{n-1} ;\left(\boldsymbol{u}_{h}^{n}, p_{h}^{n}\right),\left(\boldsymbol{v}_{h}, q_{h}\right)\right) \\
& +\frac{\rho^{\mathrm{s}} \epsilon^{\mathrm{s}}}{\tau}\left(\boldsymbol{B}_{h}\left(\boldsymbol{u}_{h}^{n} \circ \boldsymbol{\phi}_{h}^{n}\right), \boldsymbol{B}_{h}\left(\boldsymbol{v}_{h} \circ \boldsymbol{\phi}_{h}^{n}\right)\right)_{\Sigma, h} \\
= & \frac{\rho^{\mathrm{s}} \epsilon^{\mathrm{s}}}{\tau}\left(\dot{\boldsymbol{d}}_{h}^{n-1}+\tau \partial_{\tau} \dot{\boldsymbol{d}}_{h}^{n-1}, \boldsymbol{B}_{h}\left(\boldsymbol{v}_{h} \circ \boldsymbol{\phi}_{h}^{n}\right)\right)_{\Sigma, h}-b_{h}\left(\boldsymbol{\lambda}_{h}^{n-1}, \boldsymbol{B}_{h}\left(\boldsymbol{v}_{h} \circ \boldsymbol{\phi}_{h}^{n}\right)\right)
\end{aligned}
$$

and

$$
b_{h}\left(\boldsymbol{\lambda}_{h}^{n}, \boldsymbol{w}_{h}\right)=\frac{\rho^{\mathrm{s}} \epsilon^{\mathrm{s}}}{\tau}\left(\boldsymbol{B}_{h}\left(\boldsymbol{u}_{h}^{n} \circ \boldsymbol{\phi}_{h}^{n}\right)-\dot{\boldsymbol{d}}_{h}^{n-1}-\tau \partial_{\tau} \dot{\boldsymbol{d}}_{h}^{n-1}, \boldsymbol{w}_{h}\right)_{\Sigma, h}+b_{h}\left(\boldsymbol{\lambda}_{h}^{n-1}, \boldsymbol{w}_{h}\right)
$$

for $n \geq 2$. The advantage of these expressions is that, since the solid elastic term has been eliminated, only solid velocities need to be transferred from the solid to the fluid (as in a standard Dirichlet-Neumann loosely coupled scheme). The resulting solution procedure is detailed in Algorithm 4.3.

Remark 4.8. It should be noted that Algorithm 4.3 requires $\boldsymbol{\lambda}_{h}^{1}, \dot{\boldsymbol{d}}_{h}^{1}$ as initial conditions, which can be obtained by performing the first step of (4.14)-(4.16). In the particular case in which $\boldsymbol{d}_{h}^{0}=\dot{\boldsymbol{d}}_{h}^{0}=\mathbf{0}$, we can start the time-stepping directly with Algorithm 4.3 for $n \geq 1$.

The computer implementation of Algorithm 4.3 is straightforward within a standard finite element library. The algebraic formulation of the steps $2-4$ are briefly discussed in the next paragraph.

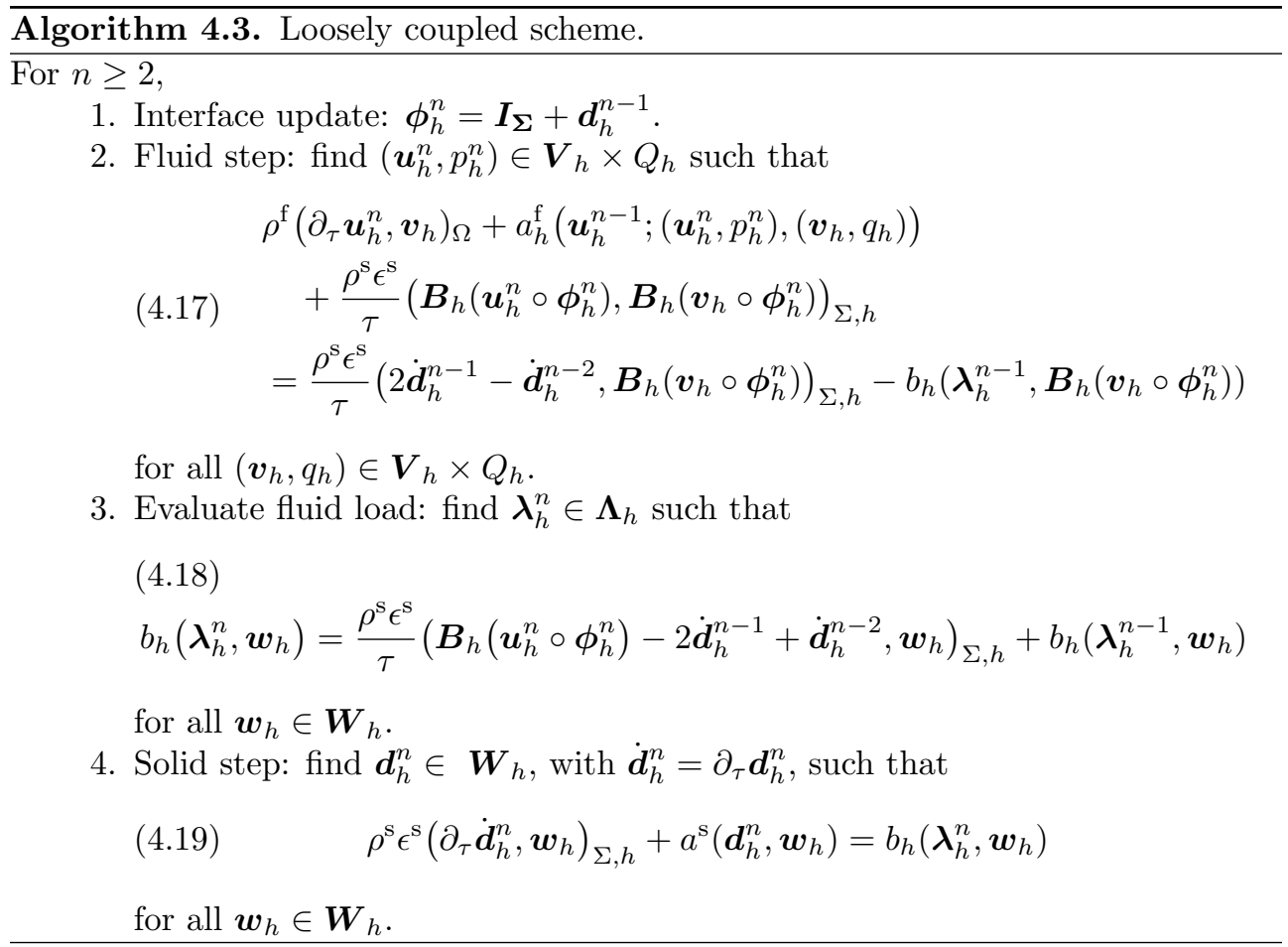


4.2. Computer implementation. Let $\mathrm{u}^{n}, \mathrm{p}^{n}, \mathrm{~d}^{n}, \dot{\mathrm{d}}^{n}, \lambda^{n}$, and $\phi^{n}$ denote the arrays of degrees of freedom associated with $\boldsymbol{u}_{h}^{n}, p_{h}^{n}, \boldsymbol{d}_{h}^{n}, \dot{\boldsymbol{d}}_{h}^{n}, \boldsymbol{\lambda}_{h}^{n}$, and $\boldsymbol{\phi}^{n}$, respectively. We also denote by $\mathrm{x}$ the array of coordinates of the points of the triangulation $\mathcal{T}_{h}^{\mathrm{s}}$. For the sake of clarity, we first consider the separated solution of the fluid without the coupling with the immersed solid. This yields the following type of linear system at each time-step:

$$
\left[\begin{array}{cc}
\mathrm{A}^{\mathrm{f}} & \mathrm{C} \\
-\mathrm{C} & \mathrm{S}
\end{array}\right]\left[\begin{array}{l}
\mathrm{u}^{n} \\
\mathrm{p}^{n}
\end{array}\right]=\left[\begin{array}{c}
\mathrm{b}^{n-1} \\
0
\end{array}\right]
$$

with the notation

$$
\mathrm{A}^{\mathrm{f}} \stackrel{\text { def }}{=} \frac{\rho^{\mathrm{f}}}{\tau} \mathrm{M}^{\mathrm{f}}+\mathrm{K}^{\mathrm{f}}, \quad \mathrm{b}^{n-1} \stackrel{\text { def }}{=} \frac{\rho^{\mathrm{f}}}{\tau} \mathrm{M}^{\mathrm{f}} \mathrm{u}^{n-1} .
$$

Here, the matrices $\mathrm{M}^{\mathrm{f}}$ and $\left[{ }_{-\mathrm{C}}^{\mathrm{K}} \mathrm{S}_{\mathrm{S}}^{\mathrm{C}}\right]$ denote the algebraic counterpart of the bilinear forms $\left(\boldsymbol{u}_{h}, \boldsymbol{v}_{h}^{\mathrm{f}}\right)$ and $a_{h}^{\mathrm{f}}\left(\boldsymbol{u}_{h}^{n-1} ;\left(\boldsymbol{u}_{h}, \boldsymbol{v}_{h}\right),\left(\boldsymbol{v}_{h}^{\mathrm{f}}, q_{h}\right)\right)$, respectively. Similarly, without interaction with the fluid, we get, for the solid, the following linear system

$$
\mathrm{A}^{\mathrm{s}} \mathrm{d}^{n}=\mathrm{r}^{n-1}
$$

with

$$
\mathrm{A}^{\mathrm{s}} \stackrel{\text { def }}{=} \frac{\rho^{\mathrm{s}} \epsilon^{\mathrm{s}}}{\tau^{2}} \mathrm{M}^{\mathrm{s}}+\mathrm{K}^{\mathrm{e}}, \quad \mathrm{r}^{n-1} \stackrel{\text { def }}{=} \frac{\rho^{\mathrm{s}} \epsilon^{\mathrm{s}}}{\tau^{2}} \mathrm{M}^{\mathrm{s}}\left(\mathrm{d}^{n-1}+\tau \dot{\mathrm{d}}^{n-1}\right), \quad \dot{\mathrm{d}}^{n}=\partial_{\tau} \mathrm{d}^{n} .
$$

Here, the matrices $\mathrm{M}^{\mathrm{s}}$ and $\mathrm{K}^{\mathrm{s}}$ stand for the algebraic counterpart of the bilinear forms $\left(\dot{\boldsymbol{d}}_{h}, \boldsymbol{w}_{h}\right)_{\Sigma, h}$ and $a^{\mathrm{s}}\left(\boldsymbol{d}_{h}, \boldsymbol{w}_{h}\right)$, respectively. Note that, due to the lumped mass approximation, the matrix $\mathrm{M}^{\mathrm{s}}$ is diagonal. Finally, we consider the matrices $\mathrm{B}^{n}$ and $\mathrm{R}^{n}$ introduced in the proof of Lemma 4.7 and define $\mathrm{L}^{n}$ as the fluid-to-solid vector interpolation matrix, e.g., for $d=2$ we have

$$
\mathrm{L}^{n} \stackrel{\text { def }}{=}\left[\begin{array}{cc}
\mathrm{B}^{n} & 0 \\
0 & \mathrm{~B}^{n}
\end{array}\right] .
$$

Based on all these considerations, the steps of Algorithm 4.3 can be reformulated, in an algebraic fashion, as:

1. Set:

$$
\phi^{n}=\mathrm{x}+\mathrm{d}^{n-1}
$$

and evaluate the interpolation matrix $\mathrm{B}^{n}$;

2. Solve fluid with solid inertial contributions:

$$
\left[\begin{array}{cc}
\mathrm{A}^{\mathrm{f}}+\frac{\rho^{\mathrm{s}} \epsilon^{\mathrm{s}}}{\tau} \mathrm{R}^{n} & \mathrm{C} \\
-\mathrm{C} & \mathrm{S}
\end{array}\right]\left[\begin{array}{c}
\mathrm{u}^{n} \\
\mathrm{p}^{n}
\end{array}\right]=\left[\begin{array}{c}
\mathrm{b}^{n-1} \\
0
\end{array}\right]
$$

3. Set:

$$
\lambda^{n}=\frac{\rho^{\mathrm{s}} \epsilon^{\mathrm{s}}}{\tau} \mathrm{M}^{\mathrm{s}}\left(\mathrm{L}^{n} \mathrm{u}^{n}-2 \dot{\mathrm{d}}^{n-1}+\dot{\mathrm{d}}^{n-2}\right)+\lambda^{n-1}
$$

4. Solve solid:

$$
\mathrm{A}^{\mathrm{s}} \mathrm{d}^{n}=\mathrm{r}^{n-1}+\lambda^{n} .
$$

It is worth recalling that, owing to Lemma 4.7, the matrix of the system (4.22) preserves the sparse pattern of the original fluid matrix $\mathrm{A}^{\mathrm{f}}$. 
4.3. Energy stability. In this section, we assume that $a^{\mathrm{s}}(\cdot, \cdot)$ is an inner product into $\boldsymbol{W}$. The associated solid energy norm is denoted by $\|\cdot\|_{\mathrm{s}} \stackrel{\text { def }}{=} \sqrt{a^{\mathrm{s}}(\cdot, \cdot)}$. We also introduce the discrete norm $\|\cdot\|_{\Sigma, h} \stackrel{\text { def }}{=} \sqrt{(\cdot, \cdot)_{\Sigma, h}}$. We shall consider the following discrete reconstruction of the elastic bilinear form $a^{\text {s }}$ : for all $\boldsymbol{d}_{h} \in \boldsymbol{W}_{h}$, we define $\boldsymbol{L}_{h}^{\mathrm{s}} \boldsymbol{d}_{h} \in \boldsymbol{W}_{h}$ such that

$$
\left(\boldsymbol{L}_{h}^{\mathrm{s}} \boldsymbol{d}_{h}, \boldsymbol{w}_{h}\right)_{\Sigma, h}=a^{\mathrm{s}}\left(\boldsymbol{d}_{h}, \boldsymbol{w}_{h}\right)
$$

for all $\boldsymbol{w}_{h} \in \boldsymbol{W}_{h}$. Furthermore, we define the discrete energy at the time-step $n \geq 0$ as

$$
E^{n}=\frac{\rho^{\mathrm{f}}}{2}\left\|\boldsymbol{u}_{h}^{n}\right\|_{0, \Omega}^{2}+\frac{\rho^{\mathrm{s}} \epsilon^{\mathrm{s}}}{2}\left\|\dot{\boldsymbol{d}}_{h}^{n}\right\|_{0, \Sigma}^{2}+\frac{1}{2}\left\|\boldsymbol{d}_{h}^{n}\right\|_{\mathrm{s}}^{2} .
$$

We will use the symbol $\lesssim$ to indicate an inequality written up to a multiplicative constant (independent of the physical and discretization parameters).

The next result establishes the unconditional energy stability of Algorithm 4.3.

TheOREM 4.9. Let $\left\{\left(\boldsymbol{u}_{h}^{n}, p_{h}^{n}, \dot{\boldsymbol{d}}_{h}^{n}, \boldsymbol{d}_{h}^{n}\right)\right\}_{n \geq 1}$ be given by Algorithm 4.3, initialized as in Remark 4.8. The following energy estimate holds for $n \geq 1$ :

$$
E^{n} \lesssim E^{0}+\tau^{2}\left\|\dot{\boldsymbol{d}}_{h}^{0}\right\|_{\mathrm{s}}^{2}+\frac{\tau^{2}}{\rho^{\mathrm{s}} \epsilon^{\mathrm{s}}}\left\|\boldsymbol{L}_{h}^{\mathrm{s}} \boldsymbol{d}_{h}^{0}\right\|_{0, \Sigma}^{2}
$$

Proof. From (4.15)-(4.16), we have

$$
\frac{\rho^{\mathrm{s}} \epsilon^{\mathrm{s}}}{\tau}\left(\dot{\boldsymbol{d}}_{h}^{n}-\boldsymbol{B}_{h}\left(\boldsymbol{u}_{h}^{n} \circ \phi_{h}^{n}\right), \boldsymbol{w}_{h}\right)_{\Sigma, h}+a^{\mathrm{s}}\left(\boldsymbol{d}_{h}^{n}-\boldsymbol{d}_{h}^{n-1}, \boldsymbol{w}_{h}\right)=0
$$

for all $\boldsymbol{w}_{h} \in \boldsymbol{W}_{h}$. In particular, owing to (4.23), we have

$$
\boldsymbol{B}_{h}\left(\boldsymbol{u}_{h}^{n} \circ \phi_{h}^{n}\right)=\dot{\boldsymbol{d}}_{h}^{n}+\frac{\tau}{\rho^{\mathrm{s}} \epsilon^{\mathrm{s}}} \boldsymbol{L}_{h}^{\mathrm{s}}\left(\boldsymbol{d}_{h}^{n}-\boldsymbol{d}_{h}^{n-1}\right) .
$$

On the other hand, by taking $\boldsymbol{w}_{h}=\boldsymbol{B}_{h}\left(\boldsymbol{v}_{h} \circ \boldsymbol{\phi}_{h}^{n}\right)$ in (4.25) and by adding the resulting expression to (4.14), we get

$$
\begin{aligned}
\rho^{\mathrm{f}}\left(\partial_{\tau} \boldsymbol{u}_{h}^{n}, \boldsymbol{v}_{h}\right)_{\Omega} & +a_{h}^{\mathrm{f}}\left(\boldsymbol{u}_{h}^{n-1} ;\left(\boldsymbol{u}_{h}^{n}, p_{h}^{n}\right),\left(\boldsymbol{v}_{h}, q_{h}\right)\right) \\
& +\rho^{\mathrm{s}} \epsilon^{\mathrm{s}}\left(\partial_{\tau} \dot{\boldsymbol{d}}_{h}^{n}, \boldsymbol{B}_{h}\left(\boldsymbol{v}_{h} \circ \boldsymbol{\phi}_{h}^{n}\right)\right)_{\Sigma, h}+a^{\mathrm{s}}\left(\boldsymbol{d}_{h}^{n}, \boldsymbol{B}_{h}\left(\boldsymbol{v}_{h} \circ \boldsymbol{\phi}_{h}^{n}\right)\right)=0
\end{aligned}
$$

for all $\left(\boldsymbol{v}_{h}, q_{h}\right) \in \boldsymbol{V}_{h} \times Q_{h}$. By taking $\left(\boldsymbol{v}_{h}, q_{h}\right)=\left(\boldsymbol{u}_{h}^{n}, p_{h}^{n}\right)$ in this expression and using the fact that

$$
a_{h}^{\mathrm{f}}\left(\boldsymbol{u}_{h}^{n-1} ;\left(\boldsymbol{u}_{h}^{n}, p_{h}^{n}\right),\left(\boldsymbol{u}_{h}^{n}, p_{h}^{n}\right)\right) \geq 2 \mu\left\|\boldsymbol{\epsilon}\left(\boldsymbol{u}_{h}^{n}\right)\right\|_{0, \Omega}^{2},
$$

we get

$$
\begin{aligned}
\frac{\rho^{\mathrm{f}}}{2} \partial_{\tau}\left\|\boldsymbol{u}_{h}^{n}\right\|_{0, \Omega}^{2} & +2 \mu\left\|\boldsymbol{\epsilon}\left(\boldsymbol{u}_{h}^{n}\right)\right\|_{0, \Omega}^{2} \\
& +\rho^{\mathrm{s}} \epsilon^{\mathrm{s}}\left(\partial_{\tau} \dot{\boldsymbol{d}}_{h}^{n}, \boldsymbol{B}_{h}\left(\boldsymbol{u}_{h}^{n} \circ \boldsymbol{\phi}_{h}^{n}\right)\right)_{\Sigma, h}+a^{\mathrm{s}}\left(\boldsymbol{d}_{h}^{n}, \boldsymbol{B}_{h}\left(\boldsymbol{u}_{h}^{n} \circ \boldsymbol{\phi}_{h}^{n}\right)\right) \leq 0 .
\end{aligned}
$$

We then proceed similarly to [20], by inserting (4.26) into (4.28). This yields

$$
\begin{array}{r}
\frac{\rho^{\mathrm{f}}}{2} \partial_{\tau}\left\|\boldsymbol{u}_{h}^{n}\right\|_{0, \Omega}^{2}+2 \mu\left\|\boldsymbol{\epsilon}\left(\boldsymbol{u}_{h}^{n}\right)\right\|_{0, \Omega}^{2}+\frac{\rho^{\mathrm{s}}}{2} \partial_{\tau}\left\|\dot{\boldsymbol{d}}_{h}^{n}\right\|_{\Sigma, h}^{2}+\frac{1}{2} \partial_{\tau}\left\|\boldsymbol{d}_{h}^{n}\right\|_{\mathrm{s}}^{2} \\
+\underbrace{\tau\left(\partial_{\tau} \dot{\boldsymbol{d}}_{h}^{n}, \boldsymbol{L}_{h}^{\mathrm{s}}\left(\boldsymbol{d}_{h}^{n}-\boldsymbol{d}_{h}^{n-1}\right)\right)_{\Sigma, h}}_{T_{1}}+\underbrace{\frac{\tau}{\rho^{\mathrm{s}} \epsilon^{\mathrm{s}}} a^{\mathrm{s}}\left(\boldsymbol{d}_{h}^{n}, \boldsymbol{L}_{h}^{\mathrm{s}}\left(\boldsymbol{d}_{h}^{n}-\boldsymbol{d}_{h}^{n-1}\right)\right)}_{T_{2}} \leq 0
\end{array}
$$


It only remains to estimate the terms $T_{1}$ and $T_{2}$. For the first term, using (4.23), we have

$$
T_{1}=\tau\left(\dot{\boldsymbol{d}}_{h}^{n}-\dot{\boldsymbol{d}}_{h}^{n-1}, \boldsymbol{L}_{h}^{\mathrm{s}} \dot{\boldsymbol{d}}_{h}^{n}\right)_{\Sigma, h}=\tau a^{\mathrm{s}}\left(\dot{\boldsymbol{d}}_{h}^{n}-\dot{\boldsymbol{d}}_{h}^{n-1}, \dot{\boldsymbol{d}}_{h}^{n}\right) \geq \frac{\tau}{2}\left(\left\|\dot{\boldsymbol{d}}_{h}^{n}\right\|_{\mathrm{s}}^{2}-\left\|\dot{\boldsymbol{d}}_{h}^{n-1}\right\|_{\mathrm{s}}^{2}\right) .
$$

Finally, for the last term, we have

$$
T_{2}=\frac{\tau}{\rho^{\mathrm{s}} \epsilon^{\mathrm{s}}}\left(\boldsymbol{L}_{h}^{\mathrm{s}} \boldsymbol{d}_{h}^{n}, \boldsymbol{L}_{h}^{\mathrm{s}}\left(\boldsymbol{d}_{h}^{n}-\boldsymbol{d}_{h}^{n-1}\right)\right)_{\Sigma, h} \geq \frac{\tau}{2 \rho^{\mathrm{s}} \epsilon^{\mathrm{s}}}\left(\left\|\boldsymbol{L}_{h}^{\mathrm{s}} \boldsymbol{d}_{h}^{n}\right\|_{\Sigma, h}^{2}-\left\|\boldsymbol{L}_{h}^{\mathrm{s}} \boldsymbol{d}_{h}^{n-1}\right\|_{\Sigma, h}^{2}\right) .
$$

We conclude by inserting the above two bounds into (4.29), by multiplying the resulting expression by $\tau$, by summing over $n$ and by applying the norms equivalence between $\|\cdot\|_{0, \Sigma}$ and $\|\cdot\|_{\Sigma, h}$ in $\boldsymbol{W}_{h}$, uniformly in $h$ (see, e.g., [48, Chapter 15]). This completes the proof.

5. Numerical experiments. The purpose of this section is to illustrate the performance of Algorithm 4.3 via comparisons with the results provided by Algorithm 4.1 (with the regularized kinematic condition (4.2)) and by an alternative method recently reported in the literature (see $[1$, Algorithm 4]). As the core motivation of the present work is the efficient simulation of heart valves, two representative two-dimensional examples which mimic the behavior of such systems in the open and closed configurations, have been considered.

In what follows, a nonlinear Reissner-Mindlin beam model is considered for the solid. Its spatial discretization is based on linear MITC (mixed interpolation of tensorial components) elements, involving two displacements and one rotation as degrees of freedom per node in the increments (see, e.g., [7]).

5.1. Idealized valve without contact. The first example is the heart-valveinspired benchmark problem considered in $[29,32,50,34,12]$. It consists of one idealized valve modeled by a cantilevered elastic beam immersed in a two dimensional channel filled with an incompressible Newtonian fluid, as shown in Figure 3. The geometry of the fluid domain is given by $\Omega=[0,8] \times[0,0.805]$. The reference configuration of the solid, $\Sigma$, is given by the segment whose endpoints are $A_{0}=(2,0)$ and $\mathrm{A}_{1}=(2,0.7)$ (see Figure 3). The physical parameters are, for the fluid, $\rho^{\mathrm{f}}=100$, $\mu=10$, and, for the solid, $\rho^{\mathrm{s}}=100, \epsilon^{\mathrm{s}}=0.0212$, with Young's modulus $E=5.6 \cdot 10^{7}$ and Poisson's ratio $\nu=0.4$.

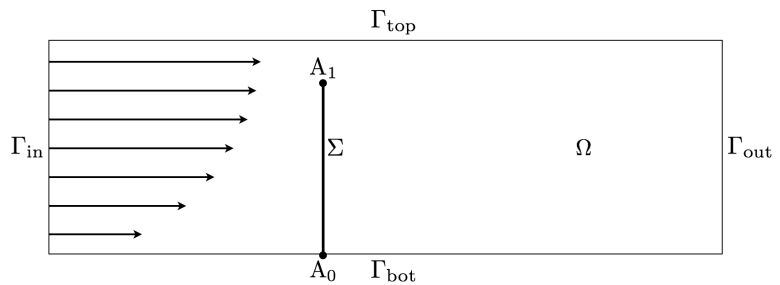

FIG. 3. Geometric configuration of the first numerical example.

A no-slip boundary condition is enforced on $\Gamma_{\text {bot }}$, and a symmetry boundary condition is imposed on $\Gamma_{\text {top }}$. Zero traction is enforced on the lateral boundary $\Gamma_{\text {out }}$, and the velocity is prescribed on $\Gamma_{\mathrm{in}}$, as a half parabolic profile whose maximum amplitude is defined by a positive time-dependent function $u_{\max }(t)$, given by the following expression:

$$
u_{\max }(t)=5(0.805)^{2}(\sin (2 \pi t)+1.1), \quad t \in \mathbb{R}^{+} .
$$


The solid is fully clamped at its bottom endpoint $\mathrm{A}_{0}$. Both the fluid and the solid are initially at rest. Considering the channel width of 0.805 as the characteristic length scale and the peak in flow speed of 6.8 as the characteristic flow speed, the associated Reynolds number is about 55 .

In this first numerical example, in which no enclosed fluid is involved, we have observed that the grad-div stabilization has pratically no impact on the quality of the numerical results. Hence, the free stabilization parameters in (3.5) have been set to $\lambda_{\mathrm{M}}=1$ and $\lambda_{\mathrm{C}}=0$ in both methods. The penalty parameter $\varepsilon$ for Algorithm 4.1 in (4.2) is set to $\varepsilon=10^{-5}$ (see [12]). We recall that Algorithm 4.3 does not involve any penalty parameter.

From the perturbed kinematic relation (4.26), Algorithms 4.1 and 4.3 are expected to deliver similar accuracy (up to the penalty error induced by $\varepsilon$ in $(4.2)$ ) when the time-step length $\tau$ is sufficiently small. Hence, we propose to compare the results provided by these two methods, using three levels of time-step refinement given by $\tau \in\left\{\left(10^{-3} / 2^{i}\right)\right\}_{i=0}^{2}$, and a fixed discretization in space based on a fluid mesh of 16,384 triangles and a solid mesh of 64 segments (see Figure 4). Figure 5a, 5c, and 5e present, respectively, for $i=\{0,1,2\}$, the comparison of the time history of the $x$-displacement of the solid at the upper tip $\mathrm{A}_{1}$, obtained with Algorithms 4.1 and 4.3. Very close results are already obtained with the largest time-step $\tau=10^{-3}$ (see Figure 5a). The agreement still improves when the time-step is refined. Note that the two curves become practically indistinguishable for the finest time grid $\tau=2.5 \cdot 10^{-4}$ (see Figure 5e). Similar observations can be made from Figures 5b, 5d, and 5f, which present the results for the $y$-displacement, respectively, for $i=\{0,1,2\}$. A slight difference is observed between the two curves for the largest time-step $\tau=10^{-3}$ (see Figure $5 \mathrm{~b}$ ). Nevertheless, this discrepancy practically disappears in the next level of refinement (see Figure 5d).

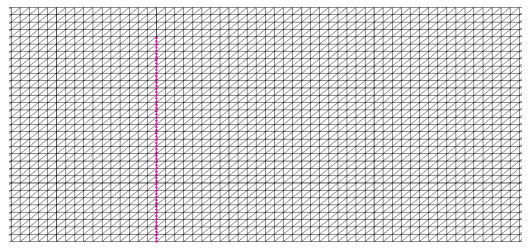

FIG. 4. Zoom on the fluid and solid meshes.

For illustration purposes we have reported in Figure 6 some snapshots of the interface location and of the fluid velocity magnitude near the solid obtained at different time instants with Algorithms 4.1 and 4.3 for $\tau=10^{-3}$. The very good agreement between both numerical approximations is noticeable. Similar observation can be inferred from the elevated pressure reported in Figure 7, obtained with $\tau=10^{-3}$. As before, Algorithm 4.3 delivers practically the same results as Algorithm 4.1, predicting the similar shape of the pressure jump across the leaflet.

Computational considerations. We finally comment on the relative efficiency of the two methods. By construction, Algorithm 4.3 requires only 1 single fluid and solid evaluations per time-step. The efficiency of Algorithm 4.1 depends on the type of solution procedure for the coupled system (4.3). In the present study, this system has been solved via a Dirichlet-Neumann interface Newton-GMRES partitioned iterative method, which requires an average of 3 fluid and solid evaluations and 21 tangent fluid and solid evaluations per time-step. Note also that the conditioning of these fluid systems is worse than in Algorithm 4.3 due to the penalized treatment of the 


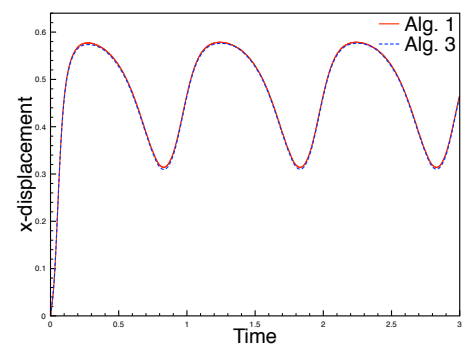

(a) $\tau=10^{-3}$.

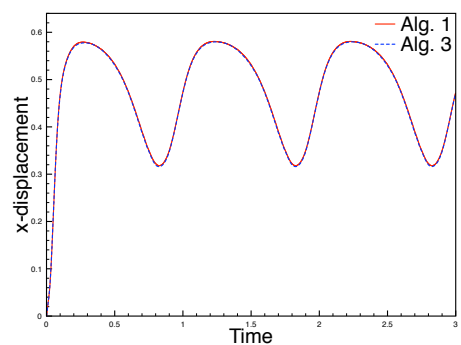

(c) $\tau=5 \cdot 10^{-4}$.

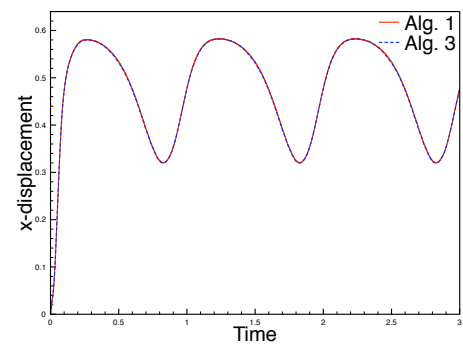

(e) $\tau=2.5 \cdot 10^{-4}$.

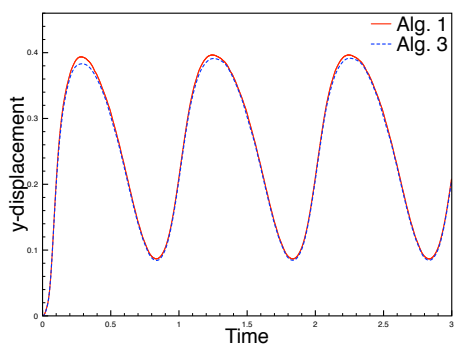

(b) $\tau=10^{-3}$.

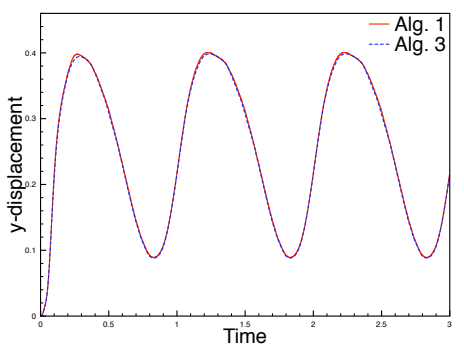

(d) $\tau=5 \cdot 10^{-4}$.

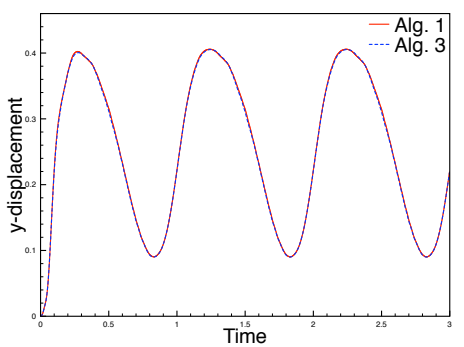

(f) $\tau=2.5 \cdot 10^{-4}$.

FIG. 5. Time history of the displacement of solid at the upper tip $A_{1}$ obtained with Algorithms 4.1 and 4.3. Left column: $x$-displacement. Right column: y-displacement.

interface coupling. This clearly points out the advantages of Algorithm 4.3 in terms of computational cost.

5.2. Idealized valve with contact. As a second example, we consider an extension of the previous one in which the idealized valve is now long enough to get into contact with the upper wall, as shown in Figure 8. The geometry of the fluid domain is given by $\Omega=[0,8] \times[0,0.805]$ as in the previous example. The reference configuration of the solid, $\Sigma$, is defined by the following analytical expression:

$$
y(x)=\frac{1}{2} \sqrt{1-\frac{(x-11 / 2)^{2}}{(3 / 2)^{2}}}, \quad x \in[4,5.112] .
$$

The coordinates of its endpoints, $\mathrm{A}_{0}$ and $\mathrm{A}_{1}$, are then $(4,0)$ and $(5.112,0.483)$, respectively. The physical parameters for the fluid are $\rho^{\mathrm{f}}=1$ and $\mu=0.03$. For the solid, we have $\rho^{\mathrm{s}}=1.2, \epsilon^{\mathrm{s}}=0.065$, with Young's modulus $E=10^{7}$ and Poisson's ratio $\nu=0.4$. 


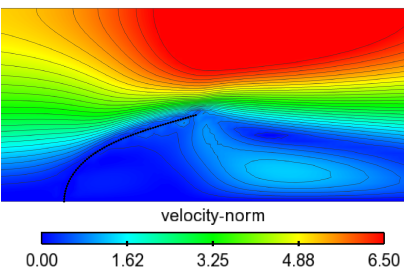

(a) Algorithm 4.1: $t=0.45$.

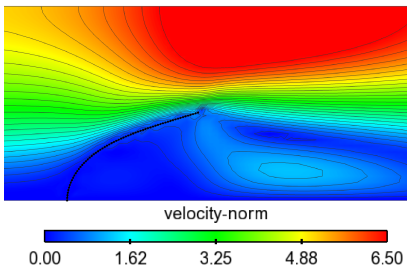

(d) Algorithm 4.3: $t=0.45$.
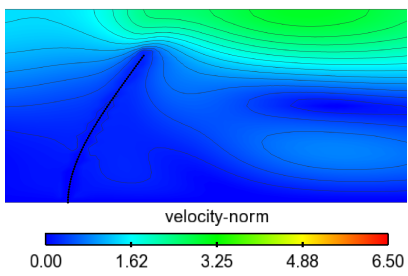

(b) Algorithm 4.1: $t=0.85$

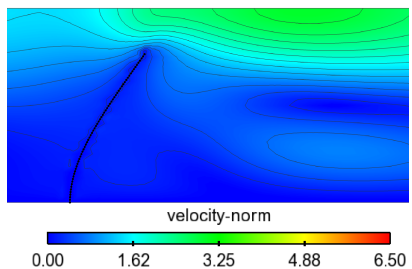

(e) Algorithm 4.3: $t=0.85$.

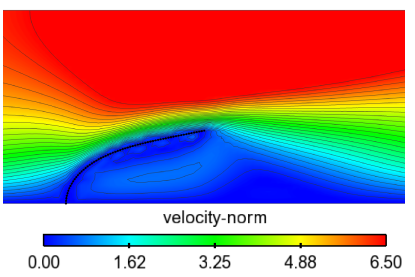

(c) Algorithm 4.1: $t=1.25$.

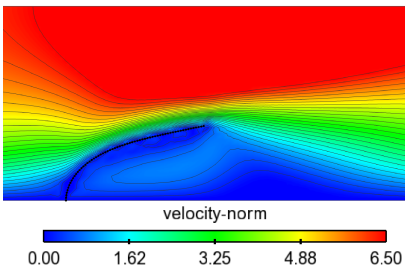

(f) Algorithm 4.3: $t=1.25$.

FIG. 6. Snapshots of the fluid velocity magnitude obtained with Algorithms 4.1 and 4.3.

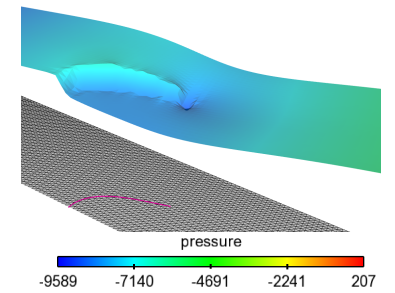

(a) Algorithm 4.1: $t=0.45$.

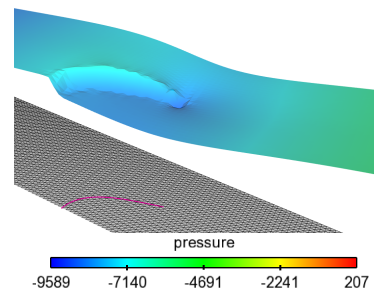

(d) Algorithm 4.3: $t=0.45$.

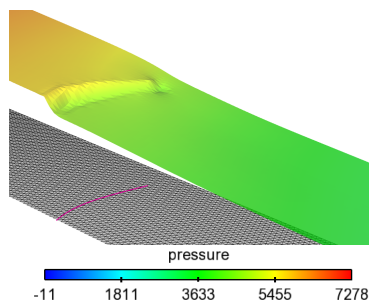

(b) Algorithm 4.1: $t=0.85$

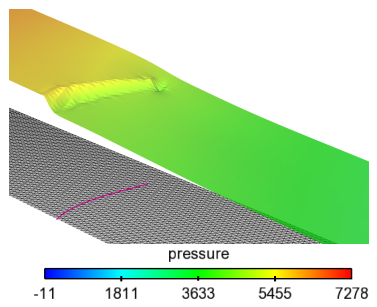

(e) Algorithm 4.3: $t=0.85$.

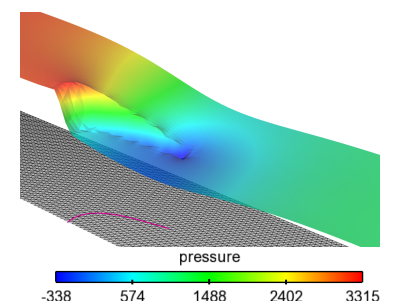

(c) Algorithm 4.1: $t=1.25$.

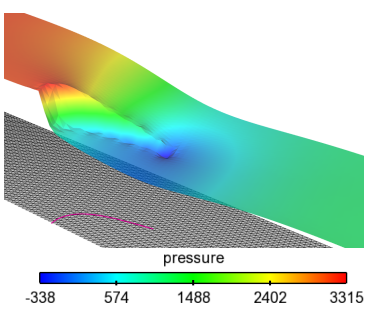

(f) Algorithm 4.3: $t=1.25$.

FIG. 7. Snapshots of the fluid elevated pressure obtained with Algorithms 4.1 and 4.3.

A no-slip boundary condition is enforced on $\Gamma_{\text {bot }}$ while a symmetry boundary condition is imposed on $\Gamma_{\text {top }}$. Zero traction is enforced on the lateral boundary $\Gamma_{\text {out }}$, while on $\Gamma_{\text {in }}$, traction is imposed in terms of the following time-dependent pressure data $p_{\text {in }}(t)$ :

$$
p_{\text {in }}(t)=\left\{\begin{aligned}
-200 \operatorname{atanh}(100 t) & \text { if } \quad 0<t<0.7, \\
200 & \text { if } \quad t \geq 0.7 .
\end{aligned}\right.
$$




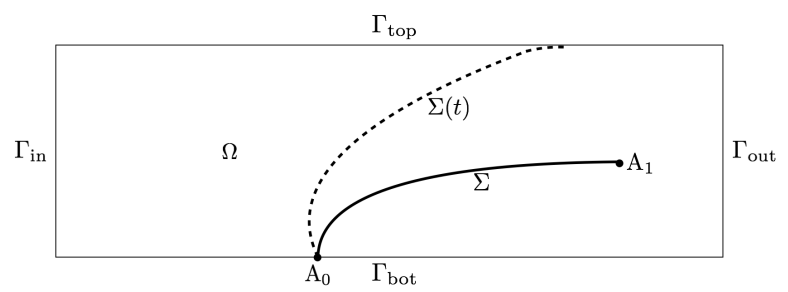

FIG. 8. Geometric configuration of the second numerical example.

The contact condition of the solid with the upper wall $\Gamma_{\text {top }}$ is the following:

$$
\boldsymbol{d} \cdot \boldsymbol{n}_{\Gamma_{\mathrm{top}}}-g \leq 0 \quad \text { on } \quad \Sigma,
$$

where $\boldsymbol{n}_{\Gamma_{\text {top }}}$ denotes the (constant) exterior unit normal to $\Gamma_{\text {top }}$ and $g: \Sigma \rightarrow \mathbb{R}^{+}$ stands for the gap function between $\Sigma$ and $\Gamma_{\text {top }}$. At the discrete level, the inequality constraint (5.2) is approximated via a penalty method (see, e.g., [46]). This amounts to include, in $a^{s}\left(\boldsymbol{d}_{h}^{n}, \boldsymbol{w}_{h}\right)$, the following additional nonlinear term

$$
\frac{\gamma_{\mathrm{c}} E \epsilon^{\mathrm{s}}}{h^{2}}\left(\left[\boldsymbol{d}_{h}^{n} \cdot \boldsymbol{n}_{\Gamma_{\mathrm{top}}}-g+\varepsilon_{h}\right]_{+}, \boldsymbol{w}_{h}\right)_{\Sigma}
$$

where $[x]_{+} \stackrel{\text { def }}{=} \max \{0, x\}, \gamma_{c}>0$ is a (dimensionless) user-defined parameter and $\varepsilon_{h}>0$ is a contact tolerance aimed at preventing penetration. In the results presented below, we have taken $\varepsilon_{h}=\mathcal{O}(h)$ and $\gamma_{c}=5 \cdot 10^{-3}$.

The fluid and the solid are initially at rest. The beam is pinched at $\mathrm{A}_{0}$ (i.e, the rotation degree of freedom is free). We consider the channel width of 0.805 as the characteristic length scale. For the characteristic flow speed, we consider the typical values of 4 and 10, for the closing and opening phases, respectively. The associated Reynolds number is then approximately 107 and 268.

Numerical evidence (not reported here) indicates that the quality of the approximations provided by Algorithm 4.1 with the regularized kinematic condition (4.2), for this specific example with contact, is extremely sensitive to the penalty parameter $\varepsilon$. In order to circumvent these difficulties, we propose to consider as the reference solution the strongly coupled Nitsche-XFEM unfitted mesh approach reported in [1, Algorithm 4]. This method has multiple interesting features (e.g., Lagrange multipliers free, consistent treatment of the interface coupling, optimal error estimates, etc.) and is known to deliver superior spatial accuracy with respect to Algorithm 4.1 (see [12]). Nevertheless, the price to pay is an increased computational complexity and a much more involved computer implementation (careful track of the interface intersections, dynamic matrix pattern, etc.) with respect to Algorithms 4.1 and 4.3.

The accuracy of Algorithm 4.3 will be then evaluated with respect to the NitscheXFEM method, by considering three successive levels of grid refinement in space and time. The coarsest level, which will be referred to as $\mathrm{M}_{1}$, corresponds to a fluid mesh of 4,096 triangles. The solid mesh is made of 25 and 50 segments, respectively, for Nitsche-XFEM and Algorithm 4.3. The corresponding time-step is set to $\tau=2 \cdot 10^{-3}$ and the contact tolerance in (5.3) to $\varepsilon_{h}=0.02$. The two subsequent space-time grids, denoted by $M_{2}$ and $M_{3}$, are uniform refinements of $M_{1}$ with, respectively, a factor of 2 and 4 along both spatial and temporal directions. The three sets of fluid and solid meshes are shown in Figure 9. For Algorithm 4.3, the value of the userdefined parameter $\varepsilon_{\mathrm{M}}$, in the SUPG/PSPG stabilization with enhanced interfacial 
mass conservation (3.6), is set to $10^{-4}$ in all levels of refinement. For the NitscheXFEM method, the user-defined parameters are set to $\gamma=100, \gamma_{\mathrm{g}}=1$ and $\gamma_{\mathrm{v}}=$ $\gamma_{\mathrm{p}}=0.01$, as detailed in $[1,12]$.

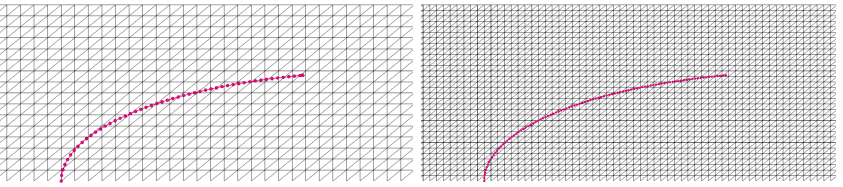

(a) $M_{1}$. (b) $\mathrm{M}_{2}$.

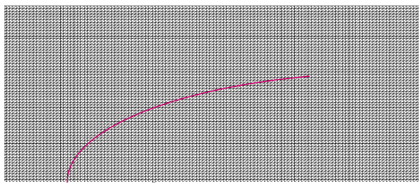

(c) $M_{3}$.

FIG. 9. Zoom on the fluid and solid meshes for the different levels of refinement.

As the negative prescribed pressure (5.1) builds up, the solid starts to bend and collides with $\Gamma_{\text {top }}$ after some time instants. Due to the flexible nature of the structure, it is free to slide or even to bounce on the wall. When contact is occurring, the fluid velocity vanishes and a pressure jump across the interface is observed. Finally, after $t=0.7$, a positive pressure builds up and the valve opens again. Figure 10 reports a comparison of the time history of the displacement of the solid at the upper tip $\mathrm{A}_{1}$ obtained with Nitsche-XFEM and Algorithm 4.3 for the three levels of refinement. The left and right columns show, respectively, the horizontal and vertical components of the displacement. Note that the flat part of the curves in the vertical displacement correspond to instants where contact occurs. The part of the curve between the two successive flat parts corresponds to the bouncing of the leaflet, illustrating the complex dynamics of the problem. A significant phase shift is observed between the two approximations for the coarsest level $\mathrm{M}_{1}$, but this discrepancy decreases with refinement. A better agreement is finally observed for the space-time grids $\mathrm{M}_{2}$ and $\mathrm{M}_{3}$.

For illustration purposes, Figure 11 presents the interface location and the fluid velocity magnitude near the leaflet obtained at $t=0.7$, for the three levels of refinement, with Nitsche-XFEM and Algorithm 4.3. Overall, a good agreement of the velocity field is already observed for the intermediate level $\mathrm{M}_{2}$ (see Figure $11 \mathrm{~b}$ and 11e). Once more, this agreement improves with space-time grid refinement as Figure $11 \mathrm{c}$ and $11 \mathrm{f}$ depict practically the same velocity field. The snapshots of the elevated pressure are given in Figure 12. The mismatch observed in Figure 10 with the coarsest approximation is clearly pointed out here in terms of the pressure jump (see Figure 12a and $12 \mathrm{~d}$ ). Note that these pressure jumps are not evaluated at the same interface location, even if evaluated at the same instant, which explains the mismatch. The situation improves via space-time grid refinement as we can infer from Figure 12c and $12 \mathrm{f}$, where the interface locations are now practically the same.

Finally, in order to provide a quantitative comparison of the two approaches, we evaluate the magnitude of the error between the two methods by measuring the $L^{2}$ difference of the interface displacement for the three levels of refinement, as shown in Table 1 . The results clearly show convergence after grid refinement.

Computational considerations. The benefits of Algorithm 4.3, with respect to the Nitsche-XFEM method considered in this example, are striking in terms of computational complexity and computer implementation. Among them, it is worth mentioning the following: there is no mesh intersection (i.e., only localization of the solid nodes within the fluid mesh are needed) and no cut-FEM (i.e., the fluid equations are integrated in the whole computational domain), the fluid system matrix has both a fixed size and a fixed standard pattern and, last but not least, the coupling scheme 


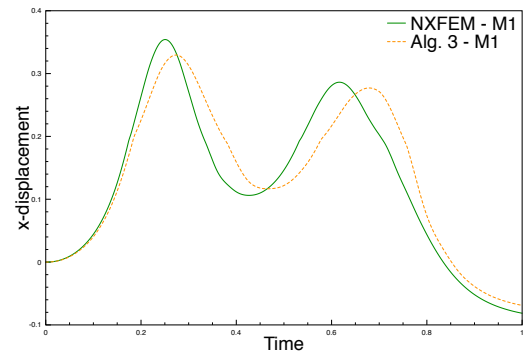

(a) $M_{1}$.

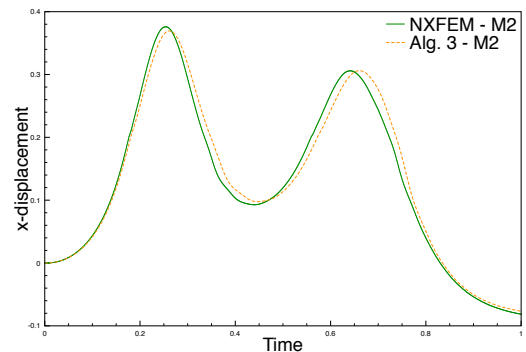

(c) $\mathrm{M}_{2}$.

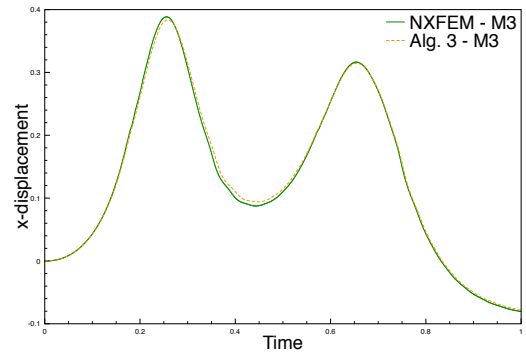

(e) $\mathrm{M}_{3}$.

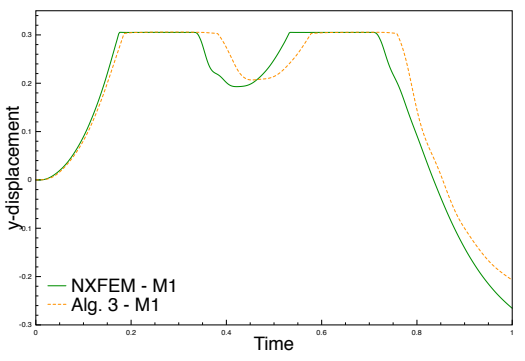

(b) $\mathrm{M}_{1}$.

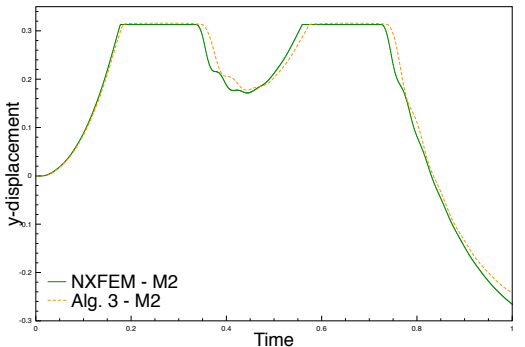

(d) $\mathrm{M}_{2}$.

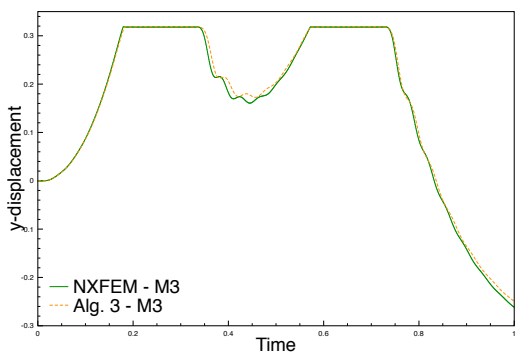

(f) $M_{3}$.

FIG. 10. Time history of the displacement of the solid at the upper tip $A_{1}$ obtained with Nitsche-XFEM and Algorithm 4.3. Left column: x-displacement. Right column: $y$-displacement.

is loosely coupled. These advantages should however be pondered carefully, since the spatial accuracy of Algorithm 4.3 relies on the use of the penalty grad-div term (3.5)-(3.6), which can compromise the efficiency of the fluid solver, particularly in three-dimensional simulations (see $[34,16,35])$.

6. Conclusions. In this paper, we have introduced a new loosely coupled scheme for the numerical approximations of incompressible fluid-structure interaction problems involving immersed thin-walled structures. The key ingredients of the proposed method are the following:

- Unfitted meshes and fictitious domain approximations in space (equivalent to a collocation method);

- Implicit treatment of the solid inertial effects within the fluid and explicit treatment of the elastic contribution;

- Lumped mass approximation in the solid. 


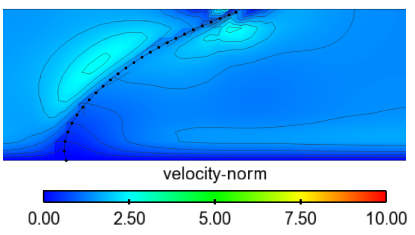

(a) Nitsche-XFEM: $M_{1}$.
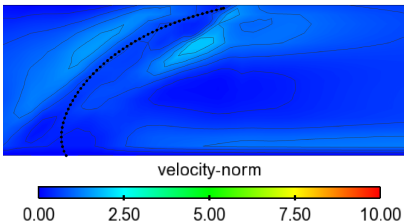

(d) Algorithm 4.3: $\mathrm{M}_{1}$.

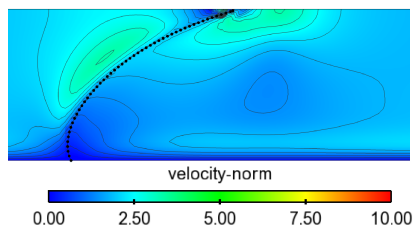

(b) Nitsche-XFEM: $M_{2}$.

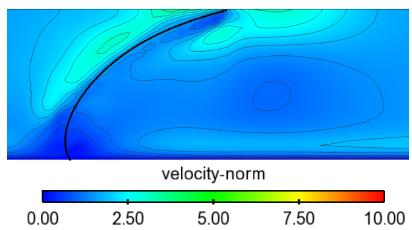

(e) Algorithm 4.3: $\mathrm{M}_{2}$.

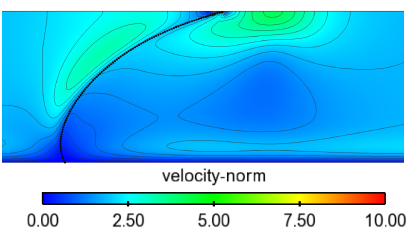

(c) Nitsche-XFEM: $M_{3}$.

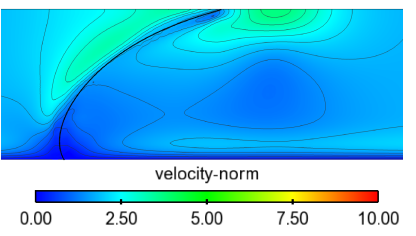

(f) Algorithm 4.3: $\mathrm{M}_{3}$.

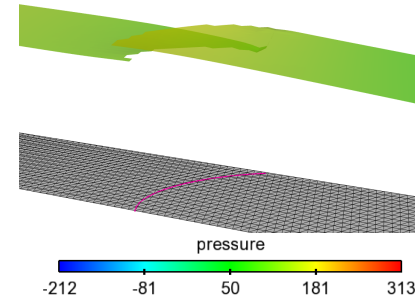

(a) Nitsche-XFEM: $M_{1}$
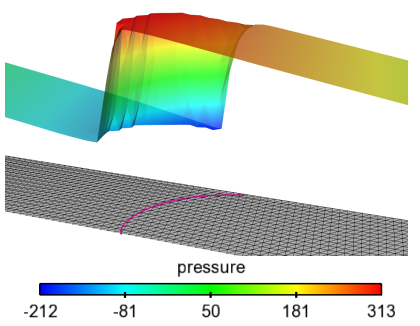

(d) Algorithm 4.3: $\mathrm{M}_{1}$.

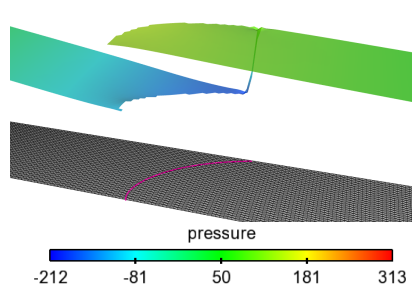

(b) Nitsche-XFEM: $\mathrm{M}_{2}$.

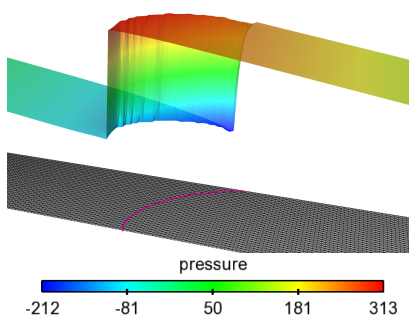

(e) Algorithm 4.3: $\mathrm{M}_{2}$

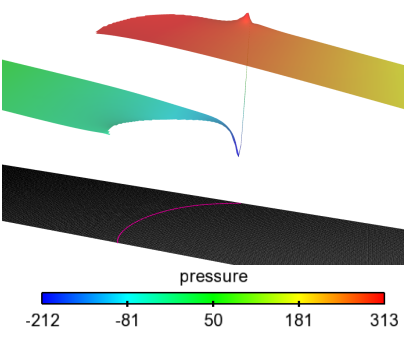

(c) Nitsche-XFEM: $M_{3}$.

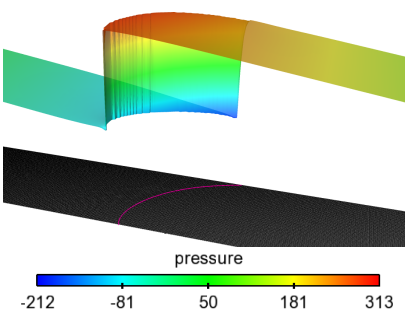

(f) Algorithm 4.3: $\mathrm{M}_{3}$.

FIG. 12. Snapshots of the fluid elevated pressure at $t=0.7$ obtained with Nitsche-XFEM and Algorithm 4.3.

TABLE 1

$L^{2}$-difference of the displacements approximations provided by Nitsche-XFEM and Algorithm 4.3.

\begin{tabular}{|c|c|}
\hline Space-time grid & $L^{2}$-difference \\
\hline $\mathrm{M}_{1}$ & $3.18 \cdot 10^{-3}$ \\
$\mathrm{M}_{2}$ & $7.48 \cdot 10^{-4}$ \\
$\mathrm{M}_{3}$ & $1.61 \cdot 10^{-5}$ \\
\hline
\end{tabular}


A salient feature of the resulting method is that it preserves both the size and the sparsity pattern of the original fluid matrix, while enabling a full splitting between the fluid and the solid time-marchings without compromising stability (Theorem 4.9). The splitting is parameter free and circumvents the usual ill-conditioning issues of fictitious domain methods involving penalized approximations of the kinematic coupling. The numerical evidence of section 5 confirmed these findings and highlighted a very good performance, in terms of accuracy and robustness, with respect to strongly coupled unfitted mesh approaches that are known to be much more computationally onerous.

The main limitation of the present numerical method comes from the spatial discretization, whose accuracy relies on a grad-div penalty term that enhances mass conservation at the expense of spoiling the conditioning of the fluid system. A forthcoming extension of this work will address the combination of the proposed loosely coupled scheme with alternative enhanced interfacial mass conservation techniques which avoid this ill-conditioning issue. Another important problem, not addressed in the present work, is the case of the coupling with immersed thick-walled solids. A first attempt in this direction could be to combine the arguments of this work with the ideas from [26, 11]. This is a particularly difficult problem, because the thick-walled nature of the solid is expected to harm the optimality of the time splitting error, as in the case of fitted meshes (see $[26,24]$ ).

\section{REFERENCES}

[1] F. Alauzet, B. Fabrèges, M. A. Fernández, and M. Landajuela, Nitsche-XFEM for the coupling of an incompressible fluid with immersed thin-walled structures, Comput. Methods Appl. Mech. Engrg., 301 (2016), pp. 300-335.

[2] M. AnNese, Time integration schemes for fluid-structure interaction problems: Non-fitted FEMs for immersed thin structures, PhD thesis, Università degli studi di Brescia, 2017.

[3] M. Astorino, J.-F. Gerbeau, O. Pantz, and K.-F. Traoré, Fluid-structure interaction and multi-body contact: Application to aortic valves, Comput. Methods Appl. Mech. Engrg., 198 (2009), pp. 3603-3612.

[4] F. BaAijens, A fictitious domain/mortar element method for fluid-structure interaction, Int. J. Numer. Methods Fluids, 35 (2001), pp. 743-761.

[5] S. Badia, A. Quaini, AND A. Quarteroni, Splitting methods based on algebraic factorization for fluid-structure interaction, SIAM J. Sci. Comput., 30 (2008), pp. 1778-1805.

[6] J. Banks, W. Henshaw, and D. Schwendeman, An analysis of a new stable partitioned algorithm for FSI problems. Part II: Incompressible flow and structural shells, J. Comput. Phys., 268 (2014), pp. 399-416.

[7] K. Bathe, Finite Element Procedures, Prentice Hall, Englewood Cliffs, NJ, 1996.

[8] F. Bertrand, P. A. Tanguy, and F. Thibault, A three-dimensional fictitious domain method for incompressible fluid flow problems, Int. J. Numer. Methods Fluids, 25 (1997), pp. 719-736.

[9] D. Boffi, N. Cavallini, And L. Gastaldi, Finite element approach to immersed boundary method with different fluid and solid densities, Math. Models Methods Appl. Sci., 21 (2011), pp. 2523-2550.

[10] D. Boffi, N. Cavallini, and L. Gastaldi, The finite element immersed boundary method with distributed Lagrange multiplier, SIAM J. Numer. Anal., 53 (2015), pp. 2584-2604.

[11] D. Boffi AND L. Gastaldi, A fictitious domain approach with lagrange multiplier for fluidstructure interactions, Numer. Math., 135 (2017), pp. 711-732.

[12] L. Boilevin-Kayl, M. A. Fernández, and J.-F. Gerbeau, Numerical methods for immersed FSI with thin-walled structures, Comput. Fluids, (2018), doi:10.1016/j.compfluid.2018.05. 024.

[13] M. Bukac, C. Canic, R. Glowinski, T. Tambaca, and A. Quaini, Fluid-structure interaction in blood flow capturing non-zero longitudinal structure displacement, J. Comp. Phys., 235 (2013), pp. 515-541.

[14] E. Burman And M. A. Fernández, Stabilization of explicit coupling in fluid-structure interaction involving fluid incompressibility, Comput. Methods Appl. Mech. Engrg., 198 (2009), pp. $766-784$. 
[15] E. Burman And M. A. Fernández, An unfitted Nitsche method for incompressible fluidstructure interaction using overlapping meshes, Comput. Methods Appl. Mech. Engrg., 279 (2014), pp. 497-514.

[16] H. Casquero, C. Bona-Casas, And H. Gomez, NURBS-based numerical proxies for red blood cells and circulating tumor cells in microscale blood flow, Comput. Methods Appl. Mech. Engrg., 316 (2017), pp. 646-667.

[17] P. Causin, J.-F. Gerbeau, and F. Nobile, Added-mass effect in the design of partitioned algorithms for fluid-structure problems, Comput. Methods Appl. Mech. Engrg., 194 (2005), pp. 4506-4527.

[18] N. Diniz dos Santos, J.-F. Gerbeau, And J.-F. Bourgat, A partitioned fluid-structure algorithm for elastic thin valves with contact, Comput. Methods Appl. Mech. Engrg., 197 (2008), pp. 1750-1761.

[19] B. Fabrèges and B. MaUry, Approximation of single layer distributions by dirac masses in finite element computations, J. Sci. Comput., 58 (2014), pp. 25-40.

[20] M. A. FERnÁNDEZ, Incremental displacement-correction schemes for incompressible fluidstructure interaction: stability and convergence analysis, Numer. Math., 123 (2013), pp. 21-65.

[21] M. A. Fernández, J. Gerbeau, and C. Grandmont, A projection semi-implicit scheme for the coupling of an elastic structure with an incompressible fluid, Int. J. Numer. Methods Engrg., 69 (2007), pp. 794-821.

[22] M. A. Fernández AND M. LANDAJUela, Splitting schemes for incompressible fluid/thin-walled structure interaction with unfitted meshes, C. R. Math. Acad. Sci. Paris, 353 (2015), pp. $647-652$.

[23] M. A. Fernández, M. Landajuela, and M. Vidrascu, Fully decoupled time-marching schemes for incompressible fluid/thin-walled structure interaction, J. Comput. Phys., 297 (2015), pp. 156-181.

[24] M. A. Fernández and J. Mullaert, Convergence and error analysis for a class of splitting schemes in incompressible fluid-structure interaction, J. Numer. Anal., 36 (2016), pp. $1748-1782$.

[25] M. A. Fernández, J. Mullaert, and M. Vidrascu, Explicit Robin-Neumann schemes for the coupling of incompressible fluids with thin-walled structures, Comput. Methods Appl. Mech. Engrg., 267 (2013), pp. 566-593.

[26] M. A. Fernández, J. Mullaert, and M. Vidrascu, Generalized Robin-Neumann explicit coupling schemes for incompressible fluid-structure interaction: Stability analysis and numerics, Int. J. Numer. Methods Engrg., 101 (2015), pp. 199-229.

[27] C. Förster, W. WAlL, And E. Ramm, Artificial added mass instabilities in sequential staggered coupling of nonlinear structures and incompressible viscous flows, Comput. Methods Appl. Mech. Engrg., 196 (2007), pp. 1278-1293.

[28] A. Gerstenberger AND W. WALL, An extended finite element method/Lagrange multiplier based approach for fluid-structure interaction, Comput. Methods Appl. Mech. Engrg., 197 (2008), pp. 1699-1714.

[29] A. J. Gil, A. Arranz Carreño, J. Bonet, and O. Hassan, An enhanced Immersed Structural Potential Method for fluid-structure interaction, J. Comput. Phys., 250 (2013), pp. 178-205.

[30] R. Glowinski, T. Pan, T. Hesla, and D. Joseph, A distributed Lagrange mutiplier/fictitious domain method for particulate flows, Int. J. Multiphase Flow, 25 (1999), pp. 755-794.

[31] G. Guidoboni, R. Glowinski, N. Cavallini, and S. Canic, Stable loosely-coupled-type algorithm for fluid-structure interaction in blood flow, J. Comp. Phys., 228 (2009), pp. 6916-6937.

[32] C. Hesch, A. J. Gil, A. Arranz Carreño, and J. Bonet, On continuum immersed strategies for Fluid-Structure Interaction, Comput. Methods Appl. Mech. Engrg., 247-248 (2012), pp. 51-64.

[33] C. Kadapa, W. Dettmer, and D. Perić, A stabilised immersed framework on hierarchical b-spline grids for fluid-flexible structure interaction with solid-solid contact, Comput. Methods Appl. Mech. Engrg., 335 (2018), pp. 472-489.

[34] D. Kamensky, M.-C. Hsu, D. Schillinger, J. Evans, A. Aggarwal, Y. Bazilevs, M. Sacks, AND T. Hughes, An immersogeometric variational framework for fluid-structure interaction: Application to bioprosthetic heart valves, Comput. Methods Appl. Mech. Engrg., 284 (2015), pp. 1005-1053.

[35] D. Kamensky, Y. Hsu, M.-C. Dnd Yu, E. J. A., M. SaCks, and T. Hughes, Immersogeometric cardiovascular fluid-structure interaction analysis with divergence-conforming B-splines, Comput. Methods Appl. Mech. Engrg., 314 (2017), pp. 408-472. 
[36] W. Kim And H. LeE, I. ChOI, A weak-coupling immersed boundary method for fluidstructure interaction with low density ratio of solid to fluid, J. Comput. Phys., 359 (2018), pp. 296-311.

[37] M. Landajuela, M. Vidrascu, D. Chapelle, and M. A. Fernández, Coupling schemes for the FSI forward predication challenge: Comparative study and validation, Int. J. Numer. Methods Biomed. Engrg., 33 (2017), e02813, 23.

[38] M. Landajuela, M. Vidrascu, D. Chapelle, and M. A. Fernández, Coupling schemes for the FSI forward prediction challenge: comparative study and validation, Int. J. Numer. Methods Biomed. Engrg., 33 (2017), e2813.

[39] P. Le TAlleC AND J. MOURO, Fluid structure interaction with large structural displacements, Comput. Methods Appl. Mech. Engrg., 190 (2001), pp. 3039-3067.

[40] A. Legay, J. Chessa, and T. Belytschko, An Eulerian-Lagrangian method for fluidstructure interaction based on level sets, Comput. Methods Appl. Mech. Engrg., 195 (2006), pp. 2070-2087.

[41] F. Nobile and C. Vergara, An effective fluid-structure interaction formulation for vascular dynamics by generalized Robin conditions, SIAM J. Sci. Comput., 30 (2008), pp. 731-763.

[42] A. PATEL, Lagrange multiplier method with penalty for elliptic and parabolic interface problems, J. Appl. Math. Comput., 37 (2011), pp. 37-56.

[43] C. Peskin, The immersed boundary method, Acta Numer., 11 (2002), pp. 479-517.

[44] A. QUAini AND A. QuARTEROni, A semi-implicit approach for fluid-structure interaction based on an algebraic fractional step method, Math. Models Methods Appl. Sci., 17 (2007), pp. 957-983.

[45] T. SAWAda And A. Tezuka, LLM and X-FEM based interface modeling of fluid-thin structure interactions on a non-interface-fitted mesh, Comput. Mech., 48 (2011), pp. 319-332.

[46] R. Scholz, Numerical solution of the obstacle problem by the penalty method, Computing, 32 (1984), pp. 297-306.

[47] T. TEZDUYAR, Stabilized finite element formulations for incompressible flow computations, in Advances in Applied Mechanics, vol. 28, Academic Press, Boston, 1992, pp. 1-44.

[48] V. ThомÉe, Galerkin finite element methods for parabolic problems, Springer Series in Computational Mathematics 25, second ed., Springer, Berlin, 2006.

[49] E. VAn Brummelen, Added mass effects of compressible and incompressible flows in fluidstructure interaction, J. Appl. Mech., 76 (2009), 021206-7.

[50] T. WICK, Flapping and contact FSI computations with the fluid-solid interfacetracking/interface-capturing technique and mesh adaptivity, Comput. Mech., 53 (2014), pp. $29-43$.

[51] A. Zilian And A. Legay, The enriched space-time finite element method (EST) for simultaneous solution of fluid-structure interaction, Int. J. Numer. Methods Engrg., 75 (2008), pp. 305-334. 